\title{
Global existence and nonlinear stability for the relativistic Vlasov-Poisson system in the gravitational case
}

\author{
Mahir Hadžić \\ Division of Applied Mathematics \\ Brown University, Providence, RI 02912, U.S.A. \\ and \\ Gerhard Rein \\ Mathematisches Institut der Universität Bayreuth \\ D 95440 Bayreuth, Germany
}

August 23, 2018

\begin{abstract}
As is well known from the work of R. Glassey and J. SchaefFER [5], the main energy estimates which are used in global existence results for the gravitational Vlasov-Poisson system do not apply to the relativistic version of this system, and smooth solutions to the initial value problem with spherically symmetric initial data of negative energy blow up in finite time. For similar reasons the variational techniques by which Y. GuO and G. REIN obtained nonlinear stability results for the Vlasov-Poisson system [6, 7, 8, 9, 18, 19] do not apply in the relativistic situation. In the present paper a direct, nonvariational approach is used to prove nonlinear stability of certain steady states of the relativistic Vlasov-Poisson system against spherically symmetric, dynamically accessible perturbations. The resulting stability estimates imply that smooth solutions with spherically symmetric initial data which are sufficiently close to the stable steady states exist globally in time.
\end{abstract}




\section{Introduction}

The topic of the present investigation is the following nonlinear system of partial differential equations, known as the relativistic Vlasov-Poisson system:

$$
\begin{gathered}
\partial_{t} f+\frac{v}{\sqrt{1+|v|^{2}}} \cdot \nabla_{x} f-\nabla_{x} U \cdot \nabla_{v} f=0, \\
\Delta U=4 \pi \rho, \lim _{|x| \rightarrow \infty} U(t, x)=0, \\
\rho(t, x)=\int f(t, x, v) d v .
\end{gathered}
$$

Here $t \in \mathbb{R}$ denotes time, $x, v \in \mathbb{R}^{3}$ denote position and momentum, and $f=f(t, x, v) \geq 0$ is the time-dependent density on phase space of a large ensemble of particles which interact only by the Newtonian gravitational potential $U=U(t, x)$ which the ensemble creates collectively through its spatial mass density $\rho=\rho(t, x)$. Collisions among the particles are assumed to be sufficiently rare to be neglected. All particles are assumed to be of the same rest mass, and all physical constants such as the rest mass of a particle, the gravitational constant, and the speed of light are normalized to unity. The individual particles obey the following equations of motion, which form the characteristic system of the Vlasov equation (1.1):

$$
\dot{x}=\frac{v}{\sqrt{1+|v|^{2}}}, \dot{v}=-\nabla_{x} U(t, x) .
$$

The system is called relativistic because of the relation between the momentum $v$ of a particle and its velocity $\dot{x}$. However, the system as a whole is neither Lorentz nor Galilei invariant.

In astrophysics galaxies or globular clusters are often modeled as large ensembles of particles, i.e., stars, in this way. But from the point of view of such applications the non-relativistic Vlasov-Poisson system where $v / \sqrt{1+|v|^{2}}$ is replaced by $v$ in the Vlasov equation is much more important, while a truly relativistic formulation is given by the Vlasov-Einstein system [1]. From a mathematics point of view the non-relativistic Vlasov-Poisson system is by now quite well understood. In particular, the initial value problem has global weak and, for smooth initial data, global classical solutions, cf. [14, 17, 23. Moreover, nonlinearly stable steady states have been established by variational techniques, i.e., by minimizing suitably chosen energy-Casimir functionals, cf. 6, 7, 8, 9, 13, 18, 19]. The stability question has also received 
a lot of attention in the astrophysics literature; we refer to [3, 4] and the references there. An extensive review of the mathematical results on global existence and stability for the non-relativistic system can be found in [20].

For the relativistic version of the system stated above both the global existence results and the variational approach to stability fail, and we briefly explain why. The total energy

$$
E_{\text {kin }}(f)+E_{\text {pot }}(f):=\iint \sqrt{1+|v|^{2}} f(x, v) d v d x-\frac{1}{8 \pi} \int\left|\nabla U_{f}(x)\right|^{2} d x
$$

of a state $f$ with induced potential $U_{f}$ is conserved along solutions, and so is the mass $\|f\|_{1}$ or any other $L^{p}$ norm $\|f\|_{p}$. Whether one wishes to minimize the energy in a variational stability analysis or to extract an apriori bound from the energy for a global existence result, in both cases one needs to control the negative potential energy in terms of a power less than one of the kinetic energy and the other conserved quantities. But the Hardy-Littlewood-Sobolev inequality together with standard interpolation arguments imply that

$$
-E_{\text {pot }}(f) \leq C\|f\|_{1}^{(2-k) / 3}\|f\|_{1+1 / k}^{(k+1) / 3} E_{\text {kin }}(f),
$$

where $0 \leq k \leq 2$, so no matter which $k$ we choose, in the context of the above estimate $E_{\text {pot }}(f)$ is of the same order as $E_{\text {kin }}(f)$. This is the reason why solutions can blow up in finite time, as shown in [5] for spherically symmetric initial data of negative energy, and why the variational stability approach fails. For the non-relativistic system $|v|^{2} / 2$ replaces $\sqrt{1+|v|^{2}}$ in the kinetic energy, and as a consequence the latter appears with the exponent $1 / 2$ in the above estimate.

Recently a non-variational stability approach was introduced for the Vlasov-Poisson system [10. Its advantage is that it can deal with steady states which are only local minimizers of an energy-Casimir functional and not global ones, in particular, it requires no lower bound on the energyCasimir functional. For the reason explained above this becomes essential in the case of the relativistic Vlasov-Poisson system, and it is the aim of the present investigation to show that this new method applies to a variety of steady states of the relativistic Vlasov-Poisson system and provides their stability against spherically symmetric, dynamically accessible perturbationsto remove the symmetry assumption is an open problem. In addition we show that the resulting stability estimates provide bounds on spherically symmetric solutions starting close to a stable steady state, which are sufficient to 
prove global existence. To our knowledge the resulting global existence results are the first such results for initial data which are not small.

The paper proceeds as follows. In the next section we state and explain our main results. Section 3 contains the stability analysis. In the Newtonian case it has been known for a long time in the astrophysics literature that the quadratic term in an expansion of a suitable energy-Casimir functional is indeed positive definite on the set of linearly dynamically accessible perturbations. We prove such a result, which can be used to study linearized stability, for the relativistic Vlasov-Poisson system. The essential difficulty is then to connect this linear result to the nonlinear problem. Here our analysis proceeds essentially as in [10, but we strive for greater generality as far as the admissible steady states are concerned, because as opposed to the Newtonian case no other stability results are available here. In Section 4 we briefly show how the stability estimates imply global existence for the corresponding, perturbed solutions. In the last section we present a large variety

of steady states to which our method applies. So far only the existence of the so-called polytropic steady states has been established for the relativistic Vlasov-Poisson system [2].

\section{Main results}

Consider a steady state $f_{0}$ of the relativistic Vlasov-Poisson system with induced potential $U_{0}$ and spatial density $\rho_{0}$, where $f_{0}$ is a function of the particle energy:

$$
f_{0}(x, v)=\phi(E) \text { where } E=E(x, v):=\sqrt{1+|v|^{2}}+U_{0}(x) .
$$

For a time-independent potential $U_{0}$ the particle energy $E$ is conserved along characteristics of the Vlasov equation so that any function of the particle energy satisfies the Vlasov equation with potential $U_{0}$, and it remains to show that for a given choice of $\phi$ the Poisson equation for $U_{0}$ has a solution, where the spatial density now becomes a functional of $U_{0}$. In Section 5 we present a variety of functions $\phi$ for which this approach gives a compactly supported steady state with finite mass. Steady states of the form (2.1) are called isotropic, and it can be shown that they are always spherically symmetric, i.e.,

$$
f_{0}(x, v)=f_{0}(A x, A v), A \in \mathrm{SO}(3), x, v \in \mathbb{R}^{3} .
$$


We want to analyze the stability of such isotropic steady states. The total energy

$$
\mathcal{H}(f):=E_{\text {kin }}(f)+E_{\text {pot }}(f)=\iint \sqrt{1+|v|^{2}} f(x, v) d v d x-\frac{1}{8 \pi} \int\left|\nabla U_{f}(x)\right|^{2} d x
$$

of a state $f$ is conserved along smooth solutions of the relativistic VlasovPoisson system. But if we expand $\mathcal{H}$ about any state $f_{0}$ with potential $U_{0}$ we find that

$$
\mathcal{H}(f)=\mathcal{H}\left(f_{0}\right)+\iint\left(\sqrt{1+|v|^{2}}+U_{0}\right)\left(f-f_{0}\right) d v d x-\frac{1}{8 \pi} \int\left|\nabla U_{f}-\nabla U_{0}\right|^{2} d x
$$

and the linear part in the expansion does not vanish. Hence we cannot use the energy as a Lyapunov function in a stability analysis. To remedy this situation we observe that for any reasonable function $\Phi$ the Casimir functional

$$
\mathcal{C}(f):=\iint \Phi(f(x, v)) d v d x
$$

is conserved as well. If we expand the energy-Casimir functional

$$
\mathcal{H}_{C}:=\mathcal{H}+\mathcal{C}
$$

about an isotropic steady state, then with $E$ defined as in Eqn. (2.1),

$$
\begin{aligned}
\mathcal{H}_{C}(f)= & \mathcal{H}_{C}\left(f_{0}\right)+\iint\left(E+\Phi^{\prime}\left(f_{0}\right)\right)\left(f-f_{0}\right) d v d x \\
& -\frac{1}{8 \pi} \int\left|\nabla U_{f}-\nabla U_{0}\right|^{2} d x+\frac{1}{2} \iint \Phi^{\prime \prime}\left(f_{0}\right)\left(f-f_{0}\right)^{2} d v d x+\ldots
\end{aligned}
$$

We want to choose $\Phi$ is such a way that at least formally $f_{0}$ is a critical point of the energy-Casimir functional, i.e., $\Phi^{\prime}\left(f_{0}\right)=-E$. In order to make this rigorous we specify the following assumptions on $\phi$ and $f_{0}$ :

Assumptions on $\phi$ and $f_{0}$ : (a) $\phi \in C(\mathbb{R})$, there exists a cut-off energy $E_{0}$ such that $\phi(E)=0$ for $E \geq E_{0}, \lim _{E \rightarrow-\infty} \phi(E)=\infty$, and $\phi \in C^{2}(]-\infty, E_{0}[)$ with

$$
\phi^{\prime}(E)<0 \text { for } E<E_{0} \text {, and } \liminf _{E \rightarrow E_{0}-} \phi^{\prime}(E)>-\infty .
$$

(b) $f_{0} \in C_{c}\left(\mathbb{R}^{6}\right)$ is compactly supported, satisfies the relation (2.1), where $U_{0}$ denotes the potential induced by $f_{0}$, and is non-trivial. 
Since

$$
\operatorname{supp} f_{0}=\left\{(x, v) \in \mathbb{R}^{6} \mid E(x, v)=\sqrt{1+|v|^{2}}+U_{0}(x) \leq E_{0}\right\}
$$

and since $U_{0}$ is spherically symmetric and strictly increasing as a function of $r=|x|$ with $\lim _{r \rightarrow \infty} U_{0}(r)=0$, part (b) necessarily implies that $E_{0}<1$. In the last section we provide a large class of steady states which satisfy these assumptions. Examples are polytropic steady states

$$
\phi(E)=\left(E_{0}-E\right)_{+}^{k},
$$

with $1 \leq k<7 / 2$, and the King model

$$
\phi(E)=\left(e^{E_{0}-E}-1\right)_{+} ;
$$

$(\cdot)_{+}$denotes the positive part.

For a function $\phi$ satisfying the assumption above, $\left.\phi:]-\infty, E_{0}\right] \rightarrow[0, \infty[$ is continuous and invertible with continuous inverse $\phi^{-1}:\left[0, \infty[\rightarrow]-\infty, E_{0}\right]$, and we define $\Phi:[0, \infty[\rightarrow \mathbb{R}$ by

$$
\Phi(f):=-\int_{0}^{f} \phi^{-1}(z) d z, f \in[0, \infty[.
$$

In particular, $\Phi \in C^{1}([0, \infty[)$.

A crucial step in the stability analysis is to specify the set of admissible perturbations. From a physics point of view perturbations arise by some exterior force field acting on the ensemble represented by the steady state $f_{0}$. Such a field induces a measure preserving flow on phase space which redistributes the particles. We refer to perturbations of the form $f=f_{0} \circ$ $T$ with $T: \mathbb{R}^{6} \rightarrow \mathbb{R}^{6}$ a measure preserving $C^{1}$-diffeomorphism as dynamically accessible from $f_{0}$. For technical reasons we have to restrict ourselves to spherically symmetric such perturbations. More precisely, we say that the $C^{1}$-diffeomorphism $T: \mathbb{R}^{6} \rightarrow \mathbb{R}^{6}$ respects spherical symmetry if for all $x, v \in \mathbb{R}^{3}$ and all rotations $A \in \mathrm{SO}(3)$,

$$
T(A x, A v)=\left(A x^{\prime}, A v^{\prime}\right) \text { and }\left|x^{\prime} \times v^{\prime}\right|=|x \times v| \text {, where }\left(x^{\prime}, v^{\prime}\right)=T(x, v) .
$$

Such a redistribution of particles on phase space would be caused by the action of a field which is spherically symmetric, and from a physics point of 
view this restriction is undesirable. The set of admissible perturbations is defined as

$$
\begin{gathered}
\mathcal{D}_{f_{0}}:=\left\{f=f_{0} \circ T \mid T: \mathbb{R}^{6} \rightarrow \mathbb{R}^{6} \text { is a measure preserving } C^{1}\right. \text {-diffeomorphism } \\
\text { which respects spherical symmetry }\} .
\end{gathered}
$$

It is important to note that this set is invariant under classical solutions of the relativistic Vlasov-Poisson system. We are going to measure the distance of a state $f \in \mathcal{D}_{f_{0}}$ from the steady state $f_{0}$ by the quantity

$$
d\left(f, f_{0}\right):=\iint\left[\Phi(f)-\Phi\left(f_{0}\right)+E\left(f-f_{0}\right)\right] d v d x+\frac{1}{8 \pi} \int\left|\nabla U_{f}-\nabla U_{0}\right|^{2} d x,
$$

which is closely related to the energy-Casimir functional:

$$
d\left(f, f_{0}\right)=\mathcal{H}_{C}(f)-\mathcal{H}_{C}\left(f_{0}\right)+\frac{1}{4 \pi} \int\left|\nabla U_{f}-\nabla U_{0}\right|^{2} d x
$$

As we will see in the next section there exists a constant $C>0$ which depends only on the steady state $f_{0}$ such that

$$
\left\|f-f_{0}\right\|_{2}^{2}+\left\|\nabla U_{f}-\nabla U_{0}\right\|_{2}^{2} \leq C d\left(f, f_{0}\right), f \in \mathcal{D}_{f_{0}} .
$$

The major part of the analysis will be concerned with proving the following result which says - in a precise, quantified manner - that the steady state is a local minimizer of the energy-Casimir functional in the set $\mathcal{D}_{f_{0}}$.

Theorem 2.1 There exist constants $\delta_{0}>0$ and $C_{0}>0$ such that for all $f \in$ $\mathcal{D}_{f_{0}}$ with $d\left(f, f_{0}\right) \leq \delta_{0}$ the following estimate holds:

$$
\mathcal{H}_{C}(f)-\mathcal{H}_{C}\left(f_{0}\right) \geq C_{0}\left\|\nabla U_{f}-\nabla U_{0}\right\|_{2}^{2}
$$

This result is proven in the next section. Our main result is the following theorem, which is an immediate corollary.

Theorem 2.2 There exist constants $\delta>0$ and $C>0$ such that for any initial datum $\stackrel{\circ}{f} \in \mathcal{D}_{f_{0}}$ with

$$
d\left(\stackrel{\circ}{f}, f_{0}\right)<\delta
$$

the corresponding solution $t \mapsto f(t)$ of the relativistic Vlasov-Poisson system with $f(0)=\stackrel{\circ}{f}$ exists globally in time and satisfies the estimate

$$
d\left(f(t), f_{0}\right) \leq C d\left(\stackrel{\circ}{f}, f_{0}\right), t \geq 0 .
$$


Proof. Let $\delta:=\delta_{0}\left(1+1 /\left(4 \pi C_{0}\right)\right)^{-1}$ with $\delta_{0}$ and $C_{0}$ from Theorem 2.1. Consider a solution $[0, T[\ni t \mapsto f(t)$ of the relativistic Vlasov-Poisson system with $\stackrel{f}{f} \mathcal{D}_{f_{0}}$ on some maximal interval of existence; as to such a local existence result we refer to the comments in Section 4. Now assume that

$$
d\left(\stackrel{\circ}{f}, f_{0}\right)<\delta<\delta_{0} .
$$

By continuity we can choose some maximal $\left.\left.t^{*} \in\right] 0, T\right]$ such that

$$
d\left(f(t), f_{0}\right)<\delta_{0}, t \in\left[0, t^{*}[.\right.
$$

Now $f(t) \in \mathcal{D}_{f_{0}}$ for all $t \in[0, T$, and hence Theorem 2.1, the relation (2.3) of $d$ to the energy-Casimir functional, and the fact that the latter is a conserved quantity yield the following chain of estimates for $t \in\left[0, t^{*}[\right.$ :

$$
\begin{aligned}
d\left(f(t), f_{0}\right) & =\mathcal{H}_{C}(f(t))-\mathcal{H}_{C}\left(f_{0}\right)+\frac{1}{4 \pi}\left\|\nabla U_{f(t)}-\nabla U_{0}\right\|_{2}^{2} \\
& \leq \mathcal{H}_{C}(f(t))-\mathcal{H}_{C}\left(f_{0}\right)+\frac{1}{4 \pi C_{0}}\left(\mathcal{H}_{C}(f(t))-\mathcal{H}_{C}\left(f_{0}\right)\right) \\
& =\left(1+\frac{1}{4 \pi C_{0}}\right)\left(\mathcal{H}_{C}(f(0))-\mathcal{H}_{C}\left(f_{0}\right)\right) \leq\left(1+\frac{1}{4 \pi C_{0}}\right) d\left(\stackrel{\circ}{f}, f_{0}\right)<\delta_{0} .
\end{aligned}
$$

This implies that $t^{*}=T$. By Proposition 4.1. $T=\infty$, and Theorem 2.2 is established.

Remark. Theorems 2.1 and 2.2 are analogous to the corresponding results obtained for the non-relativistic case in [10, but there are two differences. Firstly, the analysis in [10] is restricted to the King model, the reason being that stability for other models had already been obtained via variational methods. Since these methods do not work in the relativistic context, as was explained in the introduction, we keep the admissible steady states more general here, but the same generality would have been possible in the nonrelativistic case. Secondly, for the non-relativistic case global existence of classical solutions is known for general data, independently of any stability analysis. In the relativistic case this is not so. Theorem 2.2 includes a new global existence result for the relativistic system for data which are not subject to a size restriction. This should be compared with the results in [5], in particular, with the fact that spherically symmetric solutions with $\mathcal{H}(f)<0$ blow up in finite time. In particular, Theorem 2.2 answers an open problem which was formulated in [2, Final remarks]. 


\section{Proof of Theorem 2.1}

The essence of the proof of Theorem 2.1 is the analysis of the quadratic term

$$
D^{2} \mathcal{H}_{C}\left(f_{0}\right)[g]:=\frac{1}{2} \iint_{\left\{f_{0}>0\right\}} \Phi^{\prime \prime}\left(f_{0}\right) g^{2} d v d x-\frac{1}{8 \pi} \int\left|\nabla U_{g}\right|^{2} d x
$$

which arises in the expansion of the energy-Casimir functional $\mathcal{H}_{C}$. If Theorem 2.1 were false, then a tangent direction $g$ to the set $\mathcal{D}_{f_{0}}$ of dynamically accessible states would exist on which this quadratic part is negative, cf. Lemma 3.2. On the other hand, if we define the Poisson bracket of two functions $f, h: \mathbb{R}^{6} \rightarrow \mathbb{R}$ by

$$
\{f, h\}:=\nabla_{x} f \cdot \nabla_{v} h-\nabla_{v} f \cdot \nabla_{x} h,
$$

then for linearized, dynamically accessible states $g=\left\{f_{0}, h\right\}$ one can show that the quadratic term is indeed positive definite, cf. Lemma 3.3, we do not go into the symplectic dynamics details behind this terminology and construction. Note however that at least formally, states of this bracket form arise as tangent vectors to the manifold $\mathcal{D}_{f_{0}}$ at the point $f_{0}$, and that the set of these states is invariant under the linearized system. The analogue of Lemma 3.3 for the non-relativistic case is well known in the astrophysics literature, where it has been used to prove linearized stability [12, 16, 24], but for the relativistic case the result seems to be new. To conclude the proof of Theorem 2.1 we then show that the function $g$ with $D^{2} \mathcal{H}_{C}\left(f_{0}\right)[g] \leq 0$ obtained in Lemma 3.2 can be written in the form $g=\left\{f_{0}, h\right\}$, which contradicts Lemma 3.3. At some points the arguments are similar to the non-relativistic case considered in [10]. But since we consider a different system and also a general class of admissible steady states and not just one example, the analysis is technically more difficult, and we prefer to give a self-contained proof for the present situation.

At several points we have to exploit the spherical symmetry assumption which is part of our definition of the set $\mathcal{D}_{f_{0}}$ of dynamically accessible data. To do so we on occasion use coordinates which are adapted to the spherical symmetry:

$$
r:=|x|, w:=\frac{x \cdot v}{r}, L:=|x \times v|^{2} ;
$$

$w$ is the radial component of momentum and $L$ is the modulus of angular

momentum squared. It is easy to see that for any spherically symmetric function $f$ by abuse of notation, $f(x, v)=f(r, w, L)$. 
We first collect some properties of the function $\Phi$ defined in Eqn. (2.2).

Lemma 3.1 (a) $\Phi \in C^{1}\left(\left[0, \infty[) \cap C^{3}(] 0, \infty[), \Phi(f) \geq-E_{0} f\right.\right.$ for $f \geq 0$, and for $f>0$,

$$
\Phi^{\prime}(f)=-\phi^{-1}(f), \Phi^{\prime \prime}(f)=-\frac{1}{\phi^{\prime}\left(\phi^{-1}(f)\right)}, \Phi^{\prime \prime \prime}(f)=\frac{\phi^{\prime \prime}\left(\phi^{-1}(f)\right)}{\left(\phi^{\prime}\left(\phi^{-1}(f)\right)\right)^{3}} .
$$

(b) There exists $C>0$ such that for all functions $f \in \mathcal{D}_{f_{0}}$,

$$
\iint\left[\Phi(f)-\Phi\left(f_{0}\right)+E\left(f-f_{0}\right)\right] d v d x \geq C \iint\left|f-f_{0}\right|^{2} d v d x
$$

in particular, Eqn. 2.4 holds.

Proof. The formulas for the derivatives are obvious, and the lower bound for $\Phi$ follows from the fact that $\phi^{-1} \leq E_{0}$. The assumptions on $\phi^{\prime}$ imply that

$$
C:=\frac{1}{2} \inf \left\{\Phi^{\prime \prime}(f) \mid 0<f \leq\left\|f_{0}\right\|_{\infty}+1\right\}>0 .
$$

For $f \in \mathcal{D}_{f_{0}}$,

$$
E\left(f-f_{0}\right)=-\Phi^{\prime}\left(f_{0}\right)\left(f-f_{0}\right) \text { on }\left\{f_{0}>0\right\}
$$

while

$$
E\left(f-f_{0}\right)=E f \geq E_{0} f=-\Phi^{\prime}(0) f=-\Phi^{\prime}\left(f_{0}\right)\left(f-f_{0}\right) \text { on }\left\{f_{0}=0\right\} .
$$

Hence by Taylor's Theorem

$$
\begin{aligned}
\Phi(f)-\Phi\left(f_{0}\right)+E\left(f-f_{0}\right) & \geq \Phi(f)-\Phi\left(f_{0}\right)-\Phi^{\prime}\left(f_{0}\right)\left(f-f_{0}\right) \\
& =\lim _{\epsilon \rightarrow 0+}\left[\Phi(f+\epsilon)-\Phi\left(f_{0}+\epsilon\right)-\Phi^{\prime}\left(f_{0}+\epsilon\right)\left(f-f_{0}\right)\right] \\
& =\lim _{\epsilon \rightarrow 0+} \frac{1}{2} \Phi^{\prime \prime}\left(\xi_{\epsilon}\right)\left|f-f_{0}\right|^{2} \geq C\left|f-f_{0}\right|^{2}
\end{aligned}
$$

since $0<\xi_{\epsilon} \leq\left\|f_{0}\right\|_{\infty}+1$ as $\epsilon \rightarrow 0$, and the proof is complete.

Assuming that Theorem 2.1 were false we construct a state $g$ on which the quadratic term $D^{2} \mathcal{H}_{C}\left(f_{0}\right)$ is negative, more precisely: 
Lemma 3.2 Assume that Theorem 2.1 were false. Then there exists a function $g \in L^{2}\left(\mathbb{R}^{6}\right)$ which is spherically symmetric, supported in $\operatorname{supp} f_{0}$, even in $v$, i.e., $g(x,-v)=g(x, v)$, and such that

$$
\begin{gathered}
\frac{1}{8 \pi}\left\|\nabla U_{g}\right\|_{2}^{2}=1, \\
D^{2} \mathcal{H}_{C}\left(f_{0}\right)[g]=\frac{1}{2} \iint_{\left\{f_{0}>0\right\}} \Phi^{\prime \prime}\left(f_{0}\right) g^{2} d v d x-1 \leq 0,
\end{gathered}
$$

and for all functions $G=G(f, L) \in C^{2}\left(\left[0, \infty\left[^{2}\right)\right.\right.$ with $G(0, L)=\partial_{f} G(0, L)=0$ for $L \geq 0$ and $\partial_{f}^{2} G$ bounded,

$$
\iint \partial_{f} G\left(f_{0}, L\right) g d v d x=0
$$

Remark. Eqn. (3.6) expresses the fact that $g$ is a direction tangent to $\mathcal{D}_{f_{0}}$ at $f_{0}$. If $\tau \mapsto f(\tau)$ is a curve on $\mathcal{D}_{f_{0}}$ with $f(0)=f_{0}$ and $\frac{d}{d \tau} f(0)=g$ then by definition of $\mathcal{D}_{f_{0}}, \iint G(f(\tau), L)=\iint G\left(f_{0}, L\right)$ so that the formal derivative of the left hand side at $\tau=0$ vanishes, which is Eqn. (3.6).

Proof of Lemma [3.2. Since we assume that Theorem 2.1] is false, there exists a sequence $\left(f_{n}\right) \subset \mathcal{D}_{f_{0}}$ such that for all $n \in \mathbb{N}$,

$$
d\left(f_{n}, f_{0}\right)<\frac{1}{n}
$$

but

$$
\mathcal{H}_{C}\left(f_{n}\right)-\mathcal{H}_{C}\left(f_{0}\right)<\frac{1}{8 \pi n}\left\|\nabla U_{f_{n}}-\nabla U_{0}\right\|_{2}^{2}
$$

If we let

$$
f_{n}=f_{0}+\sigma_{n} g_{n} \text { with } \frac{1}{8 \pi}\left\|\nabla U_{g_{n}}\right\|_{2}^{2}=1
$$

i.e.,

$$
\sigma_{n}:=\frac{1}{\sqrt{8 \pi}}\left\|\nabla U_{f_{n}}-\nabla U_{0}\right\|_{2}, g_{n}:=\frac{1}{\sigma_{n}}\left(f_{n}-f_{0}\right),
$$

then in particular,

$$
\sigma_{n}^{2} \leq d\left(f_{n}, f_{0}\right)<\frac{1}{n}
$$


A weak limit $g$ of a subsequence of $\left(g_{n}\right)$.

Using the definition of $d$ and Eqns. (3.7), (3.8), and (2.3) we find that

$$
\begin{aligned}
\frac{1}{\sigma_{n}^{2}} \iint[\Phi & \left.\left(f_{n}\right)-\Phi\left(f_{0}\right)+E \sigma_{n} g_{n}\right] d v d x-1 \\
= & \frac{1}{\sigma_{n}^{2}}\left(d\left(f_{n}, f_{0}\right)-\frac{1}{4 \pi}\left\|\nabla U_{f_{n}}-\nabla U_{0}\right\|_{2}^{2}\right) \\
= & \frac{1}{\sigma_{n}^{2}}\left(\mathcal{H}_{C}\left(f_{n}\right)-\mathcal{H}_{C}\left(f_{0}\right)\right)<\frac{1}{\sigma_{n}^{2}} \frac{1}{8 \pi n}\left\|\nabla U_{f_{n}}-\nabla U_{0}\right\|_{2}^{2}=\frac{1}{n} .
\end{aligned}
$$

By Lemma 3.1 this implies that

$$
1+\frac{1}{n}>\frac{1}{\sigma_{n}^{2}} \iint\left[\Phi\left(f_{n}\right)-\Phi\left(f_{0}\right)+E \sigma_{n} g_{n}\right] d v d x \geq C \iint\left|g_{n}\right|^{2} d v d x,
$$

and hence the sequence $\left(g_{n}\right)$ is bounded in $L^{2}\left(\mathbb{R}^{6}\right)$. We extract a subsequence, again denoted by $\left(g_{n}\right)$, such that

$$
g_{n} \rightarrow g \text { weakly in } L^{2}\left(\mathbb{R}^{6}\right)
$$

Since the functions $g_{n}$ are spherically symmetric so is $g$, and we need to show that it is supported on supp $f_{0}$. The term in brackets in Eqn. (3.10) is nonnegative, and on the set $\left\{E>E_{0}\right\}$ the steady state distribution $f_{0}$ and hence also $\Phi\left(f_{0}\right)$ vanish, while $\Phi\left(f_{n}\right) \geq-E_{0} f_{n}=-E_{0} \sigma_{n} g_{n}$. Hence

$2>\frac{1}{\sigma_{n}^{2}} \iint_{\left\{E>E_{0}\right\}}\left[\Phi\left(f_{n}\right)-\Phi\left(f_{0}\right)+E \sigma_{n} g_{n}\right] d v d x \geq \frac{1}{\sigma_{n}} \iint_{\left\{E>E_{0}\right\}}\left(E-E_{0}\right) g_{n} d v d x$ and by (3.9),

$$
\iint_{\left\{E>E_{0}\right\}}\left(E-E_{0}\right) g_{n} d v d x \leq 2 \sigma_{n} \rightarrow 0, n \rightarrow \infty
$$

For any fixed $E_{0}<E_{1}<1$,

$$
\iint_{\left\{E>E_{1}\right\}} g_{n} d v d x \leq \iint_{\left\{E>E_{0}\right\}} \frac{E-E_{0}}{E_{1}-E_{0}} g_{n} d v d x \rightarrow 0 ;
$$

notice that $g_{n} \geq 0$ on the set $\left\{E>E_{0}\right\}$. Since (3.12) holds for any $E_{0}<E_{1}<1$, we conclude that $g$ is supported on $\operatorname{supp} f_{0}=\left\{E \leq E_{0}\right\}$. Moreover, since for 
$E_{0}<E_{1}<1$ fixed the set $\left\{E \leq E_{1}\right\}$ is compact, the $L^{2}$-bound on $\left(g_{n}\right)$ and Eqn. (3.12) imply that $\left(g_{n}\right)$ is bounded in $L^{1}\left(\mathbb{R}^{6}\right)$ as well.

Proof of (3.4).

First we note that $U_{0}$ is spherically symmetric and radially increasing, so in particular, $U_{0}(0) \leq U_{0} \leq 0$. Hence

$$
\begin{aligned}
\iint \sqrt{1+|v|^{2}}\left|g_{n}\right| d v d x \leq & \iint E\left|g_{n}\right| d v d x-U_{0}(0) \iint\left|g_{n}\right| d v d x \\
\leq & E_{0} \iint\left|g_{n}\right| d v d x+\iint_{\left\{E>E_{0}\right\}}\left(E-E_{0}\right)\left|g_{n}\right| d v d x \\
& -U_{0}(0) \iint\left|g_{n}\right| d v d x,
\end{aligned}
$$

so that together with (3.11) and the $L^{1}$-bound on $\left(g_{n}\right)$ the kinetic energy is bounded along $\left(g_{n}\right)$. By well known interpolation arguments [20, Ch. 1, Lemma 5.1] the sequence of induced spatial densities $\left(\rho_{g_{n}}\right)$ is bounded in $L^{5 / 4}\left(\mathbb{R}^{3}\right)$, so without loss of generality this sequence converges weakly in $L^{5 / 4}\left(\mathbb{R}^{3}\right)$. We fix $E_{0}<E_{1}<1$ and $R_{1}>0$ such that $1+U_{0}\left(R_{1}\right)=E_{1}$, which implies that $E(x, v) \geq E_{1}$ for $|x| \geq R_{1}$. Then by (3.12),

$$
\int_{\left\{|x|>R_{1}\right\}}\left|\rho_{g_{n}}\right| d x \leq \iint_{\left\{E>E_{1}\right\}} g_{n} d v d x \rightarrow 0,
$$

i.e., the sequence $\left(\rho_{g_{n}}\right)$ remains concentrated, and hence

$$
\nabla U_{g_{n}} \rightarrow \nabla U_{g} \text { strongly in } L^{2}\left(\mathbb{R}^{3}\right),
$$

cf. [20, Ch. 2, Lemma 3.2]. Passing to the limit in Eqn. (3.8) proves that $g$ satisfies the Eqn. (3.4).

Proof of (3.5).

We first claim that there exists a sequence of sets $K_{j} \subset K_{j+1} \subset \ldots \subset \operatorname{supp} f_{0}$ such that for a subsequence which we again denote by $\left(g_{n}\right)$,

$$
\operatorname{vol}\left(\operatorname{supp} f_{0} \backslash K_{j}\right)<\frac{1}{j} \text { and } \lim _{n \rightarrow \infty} \sigma_{n} g_{n}=0 \text { uniformly on } K_{j}, j \in \mathbb{N} \text {. }
$$

To see this we note that $\left\|\sigma_{n} g_{n}\right\|_{2} \leq C \sigma_{n} \rightarrow 0$ so that a subsequence converges to zero pointwise a. e.. Then we use Egorov's theorem on the set supp $f_{0}$ 
which has finite measure. In the following arguments we need to stay away from the boundary of the latter set, and hence we define

$$
S_{m}:=\left\{(x, v) \in \mathbb{R}^{6} \mid E(x, v) \leq E_{0}-1 / m\right\}, m \in \mathbb{N} .
$$

Clearly, $\delta_{m}:=\inf _{S_{m}} f_{0}>0$. Hence for all sufficiently large $n$,

$$
\delta_{m} / 2 \leq f_{0}+\sigma_{n} g_{n} \leq\left\|f_{0}\right\|_{\infty}+1 \text { on } S_{m} \cap K_{j}
$$

On the set $S_{m} \cap K_{j}$,

$$
\Phi\left(f_{n}\right)-\Phi\left(f_{0}\right)+E\left(f_{n}-f_{0}\right)=\frac{1}{2} \Phi^{\prime \prime}\left(f_{0}\right)\left(\sigma_{n} g_{n}\right)^{2}+\frac{1}{6} \Phi^{\prime \prime \prime}\left(f_{0}+\xi \sigma_{n} g_{n}\right)\left(\sigma_{n} g_{n}\right)^{3},
$$

with $0 \leq \xi \leq 1$; recall that $\Phi \in C^{3}(] 0, \infty[)$. In particular,

$$
\left|\Phi^{\prime \prime \prime}\left(f_{0}+\xi \sigma_{n} g_{n}\right)\right| \leq \sup \left\{\left|\Phi^{\prime \prime \prime}(z)\right| \mid \delta_{m} / 2 \leq z \leq\left\|f_{0}\right\|_{\infty}+1\right\}=: C_{m}<\infty .
$$

Using (3.10) we find that for all sufficiently large $n$,

$$
\begin{aligned}
\frac{1}{2} \iint_{S_{m} \cap K_{j}} \Phi^{\prime \prime}\left(f_{0}\right)\left|g_{n}\right|^{2} d v d x= & \frac{1}{\sigma_{n}^{2}} \iint_{S_{m} \cap K_{j}}\left[\Phi\left(f_{n}\right)-\Phi\left(f_{0}\right)+E\left(f_{n}-f_{0}\right)\right] d v d x \\
& -\frac{1}{6 \sigma_{n}^{2}} \iint_{S_{m} \cap K_{j}} \Phi^{\prime \prime \prime}\left(f_{0}+\xi \sigma_{n} g_{n}\right)\left(\sigma_{n} g_{n}\right)^{3} d v d x \\
< & 1+\frac{1}{n}+C_{m} \sup _{K_{j}}\left|\sigma_{n} g_{n}\right| \iint\left|g_{n}\right|^{2} d v d x .
\end{aligned}
$$

Now $g_{n} \rightarrow g$ weakly in $L^{2}\left(\mathbb{R}^{6}\right)$ and $\sigma_{n} g_{n} \rightarrow 0$ uniformly on $K_{j}$. Taking the limit $n \rightarrow \infty$ implies that for all $j, m \in \mathbb{N}$,

$$
\frac{1}{2} \iint_{S_{m} \cap K_{j}} \Phi^{\prime \prime}\left(f_{0}\right) g^{2} d v d x \leq 1
$$

the latter expression is $L^{2}$-weakly lower semicontinuous in $g$. By the boundedness of $\Phi^{\prime \prime}\left(f_{0}\right)$ on $S_{m}$ and the choice of $K_{j}$ we find with $j \rightarrow \infty$ that

$$
\frac{1}{2} \iint_{S_{m}} \Phi^{\prime \prime}\left(f_{0}\right) g^{2} d v d x \leq 1
$$

and with $m \rightarrow \infty$ the monotone convergence theorem implies Eqn. (3.5). 
Proof of (3.6).

Let $G=G(f, L)$ be a function as specified in Eqn. (3.6). By Taylor expansion with respect to the first argument,

$$
G\left(f_{n}, L\right)-G\left(f_{0}, L\right)=\partial_{f} G\left(f_{0}, L\right) \sigma_{n} g_{n}+\frac{1}{2} \partial_{f}^{2} G\left(f_{0}+\tau \sigma_{n} g_{n}, L\right)\left(\sigma_{n} g_{n}\right)^{2}
$$

for some $\tau \in[0,1]$. For $f \in \mathcal{D}_{f_{0}}, \iint G(f, L)=\iint G\left(f_{0}, L\right)$, and hence

$$
\iint \partial_{f} G\left(f_{0}, L\right) g_{n} d v d x=-\frac{1}{2} \sigma_{n} \iint \partial_{f}^{2} G\left(f_{0}+\tau \sigma_{n} g_{n}, L\right) g_{n}^{2} d v d x \rightarrow 0 ;
$$

note that $\partial_{f}^{2} G$ is bounded, $\left(g_{n}\right)$ is bounded in $L^{2}\left(\mathbb{R}^{6}\right)$, and $\sigma_{n} \rightarrow 0$. On the other hand $\partial_{f} G\left(f_{0}, L\right)$ is supported on the compact set $\operatorname{supp} f_{0}$ and hence bounded. Since $g_{n} \rightarrow g$ weakly in $L^{2}\left(\mathbb{R}^{6}\right)$, Eqn. (3.6) follows as $n \rightarrow \infty$.

Conclusion of the proof of Lemma 3.2.

The function $g$ constructed above has all the required properties, except that it need not be even in $v$. However, if we decompose it into its even and odd parts with respect to $v, g=g_{\text {even }}+g_{\text {odd }}$, then $g_{\text {even }}$ satisfies (3.4), (3.5), (3.6) as well. Since $\rho_{g}=\rho_{g_{\mathrm{even}}}$ we have $\nabla U_{g_{\mathrm{even}}}=\nabla U_{g}$, and (3.4) remains valid. Since $\Phi^{\prime \prime}\left(f_{0}\right) \geq 0$ is even in $v$,

$$
\begin{aligned}
1 & \geq \frac{1}{2} \iint_{\left\{f_{0}>0\right\}} \Phi^{\prime \prime}\left(f_{0}\right)\left(g_{\text {even }}+g_{\text {odd }}\right)^{2}=\frac{1}{2} \iint_{\left\{f_{0}>0\right\}} \Phi^{\prime \prime}\left(f_{0}\right)\left(\left(g_{\text {even }}\right)^{2}+\left(g_{\text {odd }}\right)^{2}\right) \\
& \geq \frac{1}{2} \iint_{\left\{f_{0}>0\right\}} \Phi^{\prime \prime}\left(f_{0}\right)\left(g_{\text {even }}\right)^{2},
\end{aligned}
$$

i.e., (3.5) remains valid. Finally, for $G$ as in (3.6), $\partial_{f} G\left(f_{0}, L\right)$ is even in $v$ so that the odd part of $g$ drops out of Eqn. (3.6), and the proof of Lemma 3.2 is complete.

As was said above for states of the form $g=\left\{f_{0}, h\right\}$ the quadratic term in the expansion of $\mathcal{H}_{C}$ is positive definite; the Poisson bracket was defined in Eqn. (3.2). It turns out that it will make some arguments technically easier later on, if we prove this fact for states of the form $g=\{E, h\}$.

Lemma 3.3 Let $h \in C_{c}^{\infty}\left(\mathbb{R}^{6}\right)$ be spherically symmetric with $\operatorname{supp} h \subset\left\{f_{0}>0\right\}$ and such that $h(x,-v)=-h(x, v)$. Then the following inequality holds:

$$
\begin{aligned}
& D^{2} \mathcal{H}_{C}\left(f_{0}\right)[\{E, h\}] \\
& \quad \geq-\frac{1}{2} \iint \frac{1}{\phi^{\prime}(E)}\left[|x \cdot v|^{2}\left|\left\{E, \frac{h}{x \cdot v}\right\}\right|^{2}+\frac{U_{0}^{\prime} h^{2}}{r\left(1+|v|^{2}\right)^{3 / 2}}\right] d v d x .
\end{aligned}
$$


Proof. Let

$$
U_{h}(x):=\iint \frac{\{E, h\}}{|x-y|} d v d y
$$

denote the potential induced by $-\{E, h\}$. Using the definition of the Poisson bracket,

$$
\int\{E, h\} d v=\nabla_{x} \cdot \int \frac{v}{\sqrt{1+|v|^{2}}} h(x, v) d v .
$$

Since both $h$ and $U_{h}$ are spherically symmetric,

$$
U_{h}^{\prime}(r)=4 \pi \int \frac{w}{\sqrt{1+|v|^{2}}} h(x, v) d v .
$$

By the Cauchy-Schwarz inequality,

$$
\frac{1}{8 \pi} \int\left|\nabla U_{h}\right|^{2} d x \leq 2 \pi \int\left[\int \frac{-w^{2}}{\sqrt{1+|v|^{2}}} \phi^{\prime}(E) d v\right]\left[\int \frac{1}{\sqrt{1+|v|^{2}}} \frac{-h^{2}}{\phi^{\prime}(E)} d v\right] d x .
$$

Since

$$
\frac{w^{2}}{\sqrt{1+|v|^{2}}} \phi^{\prime}(E)=w \frac{d}{d w} \phi\left(\sqrt{1+w^{2}+L / r^{2}}+U_{0}(r)\right)=w \frac{d}{d w} \phi(E),
$$

an integration by parts with respect to $w$ yields

$$
\int \frac{-w^{2}}{\sqrt{1+|v|^{2}}} \phi^{\prime}(E) d v=\rho_{0}(r):=\rho_{f_{0}}(r) .
$$

Hence

$$
D^{2} \mathcal{H}_{C}\left(f_{0}\right)[\{E, h\}] \geq-\frac{1}{2} \iint \frac{1}{\phi^{\prime}(E)}\left[|\{E, h\}|^{2}-4 \pi \frac{\rho_{0}(r)}{\sqrt{1+|v|^{2}}} h^{2}\right] d v d x .
$$

Since $h$ is odd in $v$ and thus in $w$ the function

$$
\mu(r, w, L):=\frac{1}{r w} h(r, w, L)
$$

is smooth away from $r=0$. Using the identity $h=r w \mu$ a straight forward computation shows that

$$
|\{E, h\}|^{2}=(r w)^{2}|\{E, \mu\}|^{2}+\left\{E, \mu^{2} r w\{E, r w\}\right\}-\mu^{2} r w\{E,\{E, r w\}\} .
$$


The first term is as claimed in the lemma. The second term leads to $\left\{\psi(E), \mu^{2} r w\{E, r w\}\right\}$ with $\psi$ a primitive of $1 / \phi^{\prime}$, and the integral of this expression with respect to $x$ and $v$ vanishes via an integration by parts; if we cut a small ball of radius $\epsilon$ about $x=0$ from the $x$-integral then the surface integral appearing after the integration by parts with respect to $x$ vanishes for $\epsilon \rightarrow 0$ since $r \mu^{2} \leq C / r$. Finally, another lengthy, but straight forward computation using the fact that $\Delta U_{0}=4 \pi \rho_{0}$ implies that

$$
-\mu^{2} r w\{E,\{E, r w\}\}=\frac{1}{1+|v|^{2}} \frac{h^{2}}{\sqrt{1+|v|^{2}}} \frac{U_{0}^{\prime}(r)}{r}+4 \pi \rho_{0}(r) \frac{h^{2}}{\sqrt{1+|v|^{2}}}
$$

If we substitute this into the above estimate for $D^{2} \mathcal{H}_{C}$ the proof of Lemma 3.3 is complete.

In order to derive a contradiction between Lemma 3.3 and Lemma 3.2 we have to show that the function $g$ provided by the latter can be written in the form $\{E, h\}$. Taking the opposite sign is of course equivalent but more convenient in the sequel.

Solving $g=\{-E, h\}$.

Let $g$ be the function obtained in Lemma 3.2. In the spherical variables introduced in (3.3) the equation to be solved for $h$ takes the form

$$
\frac{w}{\sqrt{1+w^{2}+L / r^{2}}} \partial_{r} h+\left(\frac{L}{r^{3} \sqrt{1+w^{2}+L / r^{2}}}-U_{0}^{\prime}(r)\right) \partial_{w} h=g .
$$

The characteristic system of this equation reads

$$
\dot{r}=\frac{w}{\sqrt{1+w^{2}+L / r^{2}}}, \quad \dot{w}=\frac{L}{r^{3} \sqrt{1+w^{2}+L / r^{2}}}-U_{0}^{\prime}(r) .
$$

In order to analyze it, we introduce for fixed $L>0$ the function

$$
\Psi_{L}(r)=U_{0}(r)+\sqrt{1+L / r^{2}}
$$

In the spherical variables the particle energy takes the form

$$
E=E(x, v)=E(r, w, L):=\sqrt{1+w^{2}+L / r^{2}}+U_{0}(r) .
$$

It is conserved along solutions of the characteristic system (3.14), while $L$ only plays the role of a parameter. For given values of $E$ and $L$ we want to 
identify the $r$-interval in which a corresponding characteristic can range. By spherical symmetry,

$$
U_{0}^{\prime}(r)=\frac{m_{0}(r)}{r^{2}}, \text { where } m_{0}(r):=4 \pi \int_{0}^{r} \rho_{0}(s) s^{2} d s, r>0 .
$$

Since the steady state is non-trivial and $U_{0}$ increasing, $U_{0}(0)<E_{0}-1$, and by (2.1), $\rho_{0}(0)>0$. Next,

$$
\Psi_{L}^{\prime}(r)=0 \Leftrightarrow m_{0}(r)-\frac{L}{\sqrt{L+r^{2}}}=0,
$$

and since the left hand side of the latter equation is strictly increasing for $L>0$ with limit $-\sqrt{L}$ for $r \rightarrow 0$ and $\left\|f_{0}\right\|_{1}>0$ for $r \rightarrow \infty$, there exists a unique radius $r_{L}>0$ such that

$$
\Psi_{L}^{\prime}\left(r_{L}\right)=0, \Psi_{L}^{\prime}(r)<0 \text { for } r<r_{L}, \Psi_{L}^{\prime}(r)>0 \text { for } r>r_{L}
$$

Since $\lim _{r \rightarrow 0} \Psi_{L}(r)=\infty$ and $\lim _{r \rightarrow \infty} \Psi_{L}(r)=1$ we conclude that for any $L>0$ and $\Psi_{L}\left(r_{L}\right)<E<1$ there exist unique radii $0<r_{-}(E, L)<r_{L}<r_{+}(E, L)<\infty$ such that

$$
\Psi_{L}\left(r_{ \pm}(E, L)\right)=E \text {, and } \Psi_{L}(r)<E \Leftrightarrow r_{-}(E, L)<r<r_{+}(E, L) .
$$

For later use we note that by the implicit function theorem the mapping ] $0, \infty\left[\ni L \mapsto r_{L}\right.$ is continuously differentiable, since $\frac{d}{d r}\left(m_{0}(r)-L / \sqrt{L+r^{2}}\right)>$ 0 . The same is true for the mapping $(E, L) \mapsto r_{ \pm}(E, L)$ on the set $\{(E, L) \in$ $\mathbb{R} \times] 0, \infty\left[\mid \Psi_{L}\left(r_{L}\right)<E<1\right\}$, since $\Psi_{L}^{\prime}(r) \neq 0$ for $r \neq r_{L}$. We also note that

$$
\Psi_{L}^{\prime \prime}\left(r_{L}\right)=4 \pi \rho_{0}\left(r_{L}\right)+\frac{L}{r_{L}\left(L+r_{L}^{2}\right)^{3 / 2}}>0 .
$$

If $h$ is to solve Eqn. (3.13), then for any solution $\tau \mapsto(r(\tau), w(\tau), L)$ of the characteristic system (3.14),

$$
\frac{d}{d \tau} h(r(\tau), w(\tau), L)=g(r(\tau), w(\tau), L) .
$$

As long as $w \neq 0$ we can rewrite this in terms of the variable $r$ :

$$
\frac{d}{d r} h(r, w(r), L)=\frac{\sqrt{1+w^{2}(r)+L / r^{2}}}{w(r)} g(r, w(r), L) .
$$


Since $E$ and $L$ are constant along characteristics we express $w$ in terms of these conserved quantities via the relation (3.15). More precisely, with

$$
w(r, E, L):=\sqrt{\left(E-U_{0}(r)\right)^{2}-L / r^{2}-1}, q(r, E, L):=\frac{E-U_{0}(r)}{w(r, E, L)}
$$

we have $w(r)=\operatorname{sign} w(r) w(r, E, L)$ and

$$
\frac{d}{d r} h(r, w(r), L)=\operatorname{sign} w(r) q(r, E, L) g(r, w(r, E, L), L) .
$$

Outside $\operatorname{supp} f_{0}$ we set $h=0$. For $(r, w, L) \in \operatorname{supp} f_{0}$ with $L>0$ we define $h$ as follows. We let $E:=E(r, w, L)$ so that $r_{-}(E, L) \leq r \leq r_{+}(E, L)$, and

$$
h(r, w, L):=\operatorname{sign} w \int_{r_{-}(E, L)}^{r} g(s, w(s, E, L), L) q(s, E, L) d s .
$$

In order for this definition to be consistent we show that the integral above vanishes if $r=r_{+}(E, L)$ so that $h(r, 0, L)=0$. To this end let $G$ be as specified in Eqn. (3.6) so that $\iint \partial_{f} G(\phi(E), L) g=0$. We want to use the change of variables $(x, v) \mapsto(r, w, L) \mapsto(r, E, L)$. Since $g$ is even in $v$ and hence in $w$ we can extend this integral only over $\{w>0\}$, and

$$
d v d x=8 \pi^{2} d r d w d L=8 \pi^{2} q(r, E, L) d r d E d L .
$$

Thus

$$
\iint_{M} \int_{r_{-}(E, L)}^{r_{+}(E, L)} g(r, w(r, E, L), L) q(r, E, L) d r \partial_{f} G(\phi(E), L) d E d L=0,
$$

where $M:=\left\{(E, L)(x, v) \mid f_{0}(x, v)>0\right\}$. The class of test functions $\partial_{f} G(\phi(E), L)$ is large enough to conclude that for almost all $E$ and $L$ the integral with respect to $r$ vanishes as desired.

As defined above the function $h$ is not sufficiently regular to apply Lemma 3.3 to it. Fubini's Theorem shows that $h$ is measurable, because by the change of variables formula the function

$$
\begin{array}{r}
(s, r, E, L) \mapsto \mathbf{1}_{\left[\Psi_{L}\left(r_{L}\right), E_{0}\right]}(E) \mathbf{1}_{\left[r_{-}(E, L), r_{+}(E, L)\right]}(s) \mathbf{1}_{[0, r]}(s) \\
g(s, w(s, E, L), L) q(s, E, L)
\end{array}
$$

is integrable; $r \leq \max \left\{|x| \mid(x, v) \in \operatorname{supp} f_{0}\right\}$, and $\mathbf{1}_{M}$ denotes the indicator function of the set $M$. 
The cut-off $h$.

We need to regularize $h$, and as a first step we study a cut-off version of $h$. To do so we need an auxiliary result.

Lemma 3.4 For every $m \in \mathbb{N}$ there exists a constant $C_{m}>0$ such that for $L \geq 1 / m$ and $\Psi_{L}\left(r_{L}\right)<E \leq E_{0}$,

$$
\int_{r_{-}(E, L)}^{r_{+}(E, L)} q(r, E, L) d r<C_{m}
$$

Proof. We first claim that for all $m \in \mathbb{N}$ there exists a constant $\eta_{m}>0$ such that for all $L \geq 1 / m, \Psi_{L}\left(r_{L}\right)<E \leq E_{0}$, and $r \in\left[r_{-}(E, L), r_{+}(E, L)\right]$,

$$
\frac{\left|\Psi_{L}^{\prime}(r)\right|}{\sqrt{\Psi_{L}(r)-\Psi_{L}\left(r_{L}\right)}} \geq \eta_{m}
$$

To see this, let $m \in \mathbb{N}$ and $L, E, r$ be as specified. Then

$$
E_{0} \geq E \geq \Psi_{L}(r)=U_{0}(r)+\sqrt{1+L / r^{2}} \geq U_{0}(0)+\sqrt{1+1 /\left(m r^{2}\right)},
$$

and hence $r \geq\left(m\left(\left(E_{0}-U_{0}(0)\right)^{2}-1\right)\right)^{-1 / 2}$. Let $R:=\max \left\{|x| \mid(x, v) \in \operatorname{supp} f_{0}\right\}$. Then

$$
L \leq r^{2}\left(\left(E_{0}-U_{0}(r)\right)^{2}-1\right) \leq R^{2}\left(\left(E_{0}-U_{0}(0)\right)^{2}-1\right) .
$$

If the above claim were false, there would exist a sequence $\left(r_{n}, L_{n}\right) \rightarrow(\bar{r}, \bar{L})$ in the set $\left[\left(m\left(\left(E_{0}-U_{0}(0)\right)^{2}-1\right)\right)^{-1 / 2}, R\right] \times\left[1 / m, R^{2}\left(\left(E_{0}-U_{0}(0)\right)^{2}-1\right)\right]$, such that

$$
\lim _{n \rightarrow \infty} \frac{\Psi_{L_{n}}^{\prime}\left(r_{n}\right)}{\sqrt{\Psi_{L_{n}}\left(r_{n}\right)-\Psi_{L_{n}}\left(r_{L_{n}}\right)}}=0
$$

We can assume that $\bar{r}=r_{\bar{L}}$ since otherwise $\Psi_{\bar{L}}(\bar{r})>\Psi_{\bar{L}}\left(r_{\bar{L}}\right)$ and $\Psi_{\bar{L}}^{\prime}(\bar{r})=0$ which is a contradiction to the uniqueness of the minimizer $r_{\bar{L}}$ of $\Psi_{\bar{L}}$. By Taylor expansion at $r=r_{L_{n}}$ we find intermediate values $\theta_{n}$ and $\tau_{n}$ between $r_{n}$ and $r_{L_{n}}$ such that

$$
\frac{\left|\Psi_{L_{n}}^{\prime}\left(r_{n}\right)\right|}{\sqrt{\Psi_{L_{n}}\left(r_{n}\right)-\Psi_{L_{n}}\left(r_{L_{n}}\right)}}=\frac{\left|\Psi_{L_{n}}^{\prime \prime}\left(\theta_{n}\right)\left(r_{n}-r_{L_{n}}\right)\right|}{\sqrt{\frac{1}{2} \Psi_{L_{n}}^{\prime \prime}\left(\tau_{n}\right)\left(r_{n}-r_{L_{n}}\right)^{2}}} \rightarrow \sqrt{2 \mid \Psi_{\bar{L}}^{\prime \prime}\left(r_{\bar{L}}\right)} \mid, n \rightarrow \infty
$$

so that (3.21) contradicts (3.16), and (3.20) is established. 
We split the integral under investigation into two parts,

$$
\int_{r_{-}(E, L)}^{r_{+}(E, L)} q(r, E, L) d r=\int_{r_{-}(E, L)}^{r_{L}} \cdots+\int_{r_{L}}^{r_{+}(E, L)} \cdots=: I_{1}+I_{2} .
$$

Using the definitions of $w(r, E, L)$ and $\Psi_{L}$ and the fact that $E-U_{0}(r) \geq$ $\Psi_{L}(r)-U_{0}(r) \geq 1$ we find that

$$
\begin{aligned}
q(r, E, L)=\frac{E-U_{0}(r)}{w(r, E, L)} & =\frac{E-U_{0}(r)}{\sqrt{\left(E-U_{0}(r)\right)^{2}-\left(\Psi_{L}(r)-U_{0}(r)\right)^{2}}} \\
& =\frac{E-U_{0}(r)}{\sqrt{\left(E-\Psi_{L}(r)\right)\left(E+\Psi_{L}(r)-2 U_{0}(r)\right)}} \\
& \leq \frac{E-U_{0}(0)}{\sqrt{2\left(E-\Psi_{L}(r)\right)}} \leq \frac{C}{\sqrt{E-\Psi_{L}(r)}}
\end{aligned}
$$

for some constant $C$ which does not depend on $r, E$, or $L$. In the integral $I_{1}$ we change variables $u=\sqrt{\Psi_{L}(r)-\Psi_{L}\left(r_{L}\right)}$ so that $\frac{d u}{d r}=\frac{1}{2 u} \Psi_{L}^{\prime}(r)<0$ on $\left[r_{-}(E, L), r_{L}[\right.$. By (3.20) and the previous estimate,

$$
\begin{aligned}
I_{1} & \leq C \int_{\sqrt{E-\Psi_{L}\left(r_{L}\right)}}^{0} \frac{1}{\sqrt{E-\Psi_{L}\left(r_{L}\right)-u^{2}}} \frac{d r}{d u} d u \\
& \leq \frac{1}{\eta_{m}} \int_{0}^{\sqrt{E-\Psi_{L}\left(r_{L}\right)}} \frac{d u}{\sqrt{E-\Psi_{L}\left(r_{L}\right)-u^{2}}}=\frac{1}{\eta_{m}} \int_{0}^{1} \frac{d s}{\sqrt{1-s^{2}}}<\infty .
\end{aligned}
$$

We argue similarly for $I_{2}$, and the proof of the lemma is complete.

For $m \in \mathbb{N}$ let

$$
\Omega_{m}:=\left\{(x, v) \in \mathbb{R}^{6} \mid E(x, v) \leq E_{0}-\frac{1}{m}, L(x, v) \geq \frac{1}{m}\right\} .
$$

With the help of the previous lemma we can prove that $\mathbf{1}_{\Omega_{m}} h$ is square integrable and solves the equation $\left\{-E, \mathbf{1}_{\Omega_{m}} h\right\}=\mathbf{1}_{\Omega_{m}} g$ in the sense of distributions.

Lemma 3.5 For $m \in \mathbb{N}$ large, $\mathbf{1}_{\Omega_{m}} h \in L^{2}\left(\mathbb{R}^{6}\right)$, and for any spherically symmetric test function $\psi=\psi(r, w, L) \in C^{1}([0, \infty[\times \mathbb{R} \times[0, \infty[)$,

$$
\iint\{-E, \psi\} \mathbf{1}_{\Omega_{m}} h d v d x=-\iint \psi \mathbf{1}_{\Omega_{m}} g d v d x
$$


Proof. In order to prove that $\mathbf{1}_{\Omega_{m}} h \in L^{2}\left(\mathbb{R}^{6}\right)$ we observe that the integrand is even in $v$ so that we can apply the change of variables (3.19):

$$
\iint \mathbf{1}_{\Omega_{m}} h^{2} d v d x=8 \pi^{2} \iint_{S_{m}} \int_{r_{-}(E, L)}^{r_{+}(E, L)} h^{2}(r, w(r, E, L), L) q(r, E, L) d r d E d L,
$$

where

$$
S_{m}:=\left\{(E, L)=(E, L)(x, v) \mid(x, v) \in \Omega_{m}\right\} .
$$

Let $(E, L) \in S_{m}$. Writing $w(r)=w(r, E, L), q(r)=q(r, E, L)$ and $r_{ \pm}=r_{ \pm}(E, L)$ for brevity the definition (3.18) of $h$, the Cauchy-Schwarz inequality, and Lemma 3.4 imply that

$$
\begin{aligned}
\int_{r_{-}}^{r_{+}} h^{2}(r, w(r), L) q(r) d r & =\int_{r_{-}}^{r_{+}}\left[\int_{r_{-}}^{r} g(s, w(s), L) q(s) d s\right]^{2} q(r) d r \\
& \leq\left(\int_{r_{-}}^{r_{+}} q(r) d r\right)^{2} \int_{r_{-}}^{r_{+}} g^{2}(r, w(r), L) q(r) d r \\
& \leq C_{m}^{2} \int_{r_{-}}^{r_{+}} g^{2}(r, w(r), L) q(r) d r
\end{aligned}
$$

A further integration with respect to $E$ and $L$ and the change of variables $(r, E, L) \mapsto(x, v)$ shows that $\left\|\mathbf{1}_{\Omega_{m}} h\right\|_{2}$ is bounded in terms of $C_{m}$ and $\|g\|_{2}$.

Let $\psi$ be a test function as specified in the lemma. As above, we parameterize the solutions of (3.14) by $r$, distinguishing between $w>0$ and $w<0$. Then it is easy to show that

$$
\{-E, \psi\}=\operatorname{sign} w \frac{1}{q(r)} \frac{d}{d r}[\psi(r, w(r), L)]
$$

Using the same change of variables as before and recalling Eqn. (3.18) we 
find that

$$
\begin{aligned}
\iint\{-E, \psi\} \mathbf{1}_{\Omega_{m}} h d v d x=\int_{\{w>0\}} \cdots+\int_{\{w<0\}} \ldots \\
=8 \pi^{2} \iint_{S_{m}} \int_{r_{-}}^{r_{+}} \frac{1}{q(r)} \frac{d}{d r}[\psi(r, w(r), L)] h(r, w(r), L) q(r) d r d E d L \\
\quad-8 \pi^{2} \iint_{S_{m}} \int_{r_{-}}^{r_{+}} \frac{1}{q(r)} \frac{d}{d r}[\psi(r,-w(r), L)] h(r,-w(r), L) q(r) d r d E d L \\
=-8 \pi^{2} \iint_{S_{m}} \int_{r_{-}}^{r_{+}} \psi(r, w(r), L) g(r, w(r), L) q(r) d r d E d L \\
\quad-8 \pi^{2} \iint_{S_{m}} \int_{r_{-}}^{r_{+}} \psi(r,-w(r), L) g(r,-w(r), L) q(r) d r d E d L \\
=-\iint \psi \mathbf{1}_{\Omega_{m}} g d v d x
\end{aligned}
$$

note that $h(r, \pm w(r), L)=0$ for $r=r_{ \pm}(E, L)$ which together with the definition of $h$ is used in the integration by parts above, and also that $g$ is even both in $v$ and $w$. The proof of Lemma 3.5 is complete.

Regularization of $h$.

The function $\mathbf{1}_{\Omega_{m}} h$ is not smooth, so in order to apply Lemma 3.3 we smooth it. For $m \in \mathbb{N}$ fixed the function $\mathbf{1}_{\Omega_{m}} h$ is, as a function of $r, w, L$, supported in a cube of the form

$$
Q:=\left[R_{0}, R_{1}\right] \times\left[-W_{0}, W_{0}\right] \times\left[L_{0}, L_{1}\right],
$$

with $0<R_{0}<R_{1}, W_{0}>0$, and $0<L_{0}<L_{1}$. Let $\zeta \in C_{c}^{\infty}\left(\mathbb{R}^{3}\right)$ be even in all three variables, i.e., $\zeta\left(z_{1}, z_{2}, z_{3}\right)=\zeta\left(\left|z_{1}\right|,\left|z_{2}\right|,\left|z_{3}\right|\right), \zeta \geq 0, \int \zeta=1$, and let $\zeta_{n}(z):=n^{3} \zeta(n z)$ for $n \in \mathbb{N}$. For $n$ sufficiently large we define

$$
h_{n}(r, w, L):=\int_{0}^{\infty} \int_{-\infty}^{\infty} \int_{0}^{\infty}\left(\mathbf{1}_{\Omega_{m}} h\right)(\bar{r}, \bar{w}, \bar{L}) \zeta_{n}(r-\bar{r}, w-\bar{w}, L-\bar{L}) d \bar{L} d \bar{w} d \bar{r} .
$$

Clearly, $h_{n} \in C_{c}^{\infty}(] 0, \infty[\times \mathbb{R} \times] 0, \infty[)$, and without loss of generality we can assume that $\operatorname{supp} h_{n} \subset Q \cap\left\{E<E_{0}\right\}$. Since $h$ is odd in $w$, so is $h_{n}$, and clearly $h_{n} \rightarrow \mathbf{1}_{\Omega_{m}} h$ in $L^{1} \cap L^{2}$. The crucial step is to show that

$$
\lim _{n \rightarrow \infty}\left\{-E, h_{n}\right\}=\mathbf{1}_{\Omega_{m}} g \text { in } L^{2} .
$$


We fix $(r, w, L)$, write $p:=\sqrt{1+w^{2}+L / r^{2}}, \bar{p}:=\sqrt{1+\bar{w}^{2}+\bar{L} / \bar{r}^{2}}$, and $\int:=$ $\int_{0}^{\infty} \int_{-\infty}^{\infty} \int_{0}^{\infty}$ for brevity, and split the convolution integral as follows:

$$
\begin{aligned}
\left\{-E, h_{n}\right\}=\frac{w}{p} \partial_{r} h_{n}+\left(\frac{L}{r^{3} p}-U_{0}^{\prime}(r)\right) \partial_{w} h_{n} & \\
= & \int\left(\mathbf{1}_{\Omega_{m}} h\right)(\bar{r}, \bar{w}, \bar{L})\left[\left(\frac{\bar{w}}{\bar{p}}-\frac{w}{p}\right) \partial_{\bar{r}}+\left(\frac{\bar{L}}{\bar{r}^{3} \bar{p}}-\frac{L}{r^{3} p}-U_{0}^{\prime}(\bar{r})+U_{0}^{\prime}(r)\right) \partial_{\bar{w}}\right] \\
& -\int\left(\mathbf{1}_{\Omega_{m}} h\right)(\bar{r}, \bar{w}, \bar{L})\left[\frac{\bar{w}}{\bar{p}} \partial_{\bar{r}}+\left(\frac{\bar{L}}{\bar{r}^{3} \bar{p}}-U_{0}^{\prime}(\bar{r})\right) \partial_{\bar{w}}\right] \\
& \left.\zeta_{n}(r-\bar{r}, w-\bar{w}, L-\bar{L}) d \bar{r} d \bar{w} d \bar{L}\right) d \bar{r} d \bar{w} d \bar{L} \\
=: & J_{1}+J_{2} .
\end{aligned}
$$

According to Lemma 3.5 .

$$
\begin{aligned}
J_{2} & =-\int \mathbf{1}_{\Omega_{m}} h\left\{-E, \zeta_{n}(r-\cdot, w-\cdot, L-\cdot)\right\} \\
& =\int\left(\mathbf{1}_{\Omega_{m}} g\right)(\bar{r}, \bar{w}, \bar{L}) \zeta_{n}(r-\bar{r}, w-\bar{w}, L-\bar{L}) d \bar{r} d \bar{w} d \bar{L} \rightarrow \mathbf{1}_{\Omega_{m}} g \text { in } L^{2} .
\end{aligned}
$$

We show that $J_{1}$ converges to 0 as $n \rightarrow \infty$. To this end we introduce new variables $\tilde{r}=n(r-\bar{r}), \tilde{w}=n(w-\bar{w}), \tilde{L}=n(L-\bar{L})$. Then $\bar{r}=r-\tilde{r} / n$, $\bar{w}=w-\tilde{w} / n, \bar{L}=L-\tilde{L} / n, d \bar{r} d \bar{w} d \bar{L}=n^{-3} d \tilde{r} d \tilde{w} d \tilde{L}$, and $\partial_{r} \zeta_{n}(r-\bar{r}, w-\bar{w}, L-$ $\bar{L})=n^{4} \partial_{\tilde{r}} \zeta(\tilde{r}, \tilde{w}, \tilde{L})$. Now we note that

$$
\begin{aligned}
\int\left(\mathbf{1}_{\Omega_{m}} h\right)(\bar{r}, \bar{w}, \bar{L}) & \left(\frac{\bar{w}}{\bar{p}}-\frac{w}{p}\right) \partial_{\bar{r}} \zeta_{n}(r-\bar{r}, w-\bar{w}, L-\bar{L}) d \bar{r} d \bar{w} d \bar{L} \\
= & n \int\left(\mathbf{1}_{\Omega_{m}} h\right)(\bar{r}, \bar{w}, \bar{L})\left(\frac{\bar{w}}{\bar{p}}-\frac{w}{p}\right) \partial_{\tilde{r}} \zeta_{n}(\tilde{r}, \tilde{w}, \tilde{L}) d \tilde{r} d \tilde{w} d \tilde{L}
\end{aligned}
$$

The analogous identity holds for the second part of $J_{1}$, and we find that

$$
\begin{aligned}
n\left(\frac{\bar{w}}{\bar{p}}-\frac{w}{p}\right) & =n \frac{\bar{w}-w}{\bar{p}}+n w\left(\frac{1}{\bar{p}}-\frac{1}{p}\right) \\
& =-\frac{\tilde{w}}{p}+n w \frac{w^{2}-\bar{w}^{2}+L / r^{2}-\bar{L} / \bar{r}^{2}}{\bar{p} p(\bar{p}+p)} \\
& \rightarrow-\frac{\tilde{w}}{p}+\frac{2 \tilde{w} w^{2}+w \tilde{L} / r^{2}-2 \tilde{r} w L / r^{3}}{2 p^{3}},
\end{aligned}
$$


since

$$
\frac{L}{r^{2}}-\frac{\bar{L}}{\bar{r}^{2}}=\frac{L-\bar{L}}{\bar{r}^{2}}+L \frac{(\bar{r}-r)(\bar{r}+r)}{r^{2} \bar{r}^{2}}
$$

We easily see that

$$
n\left(U_{0}^{\prime}(r)-U_{0}^{\prime}(\bar{r})\right) \rightarrow U_{0}^{\prime \prime}(r) \tilde{r}
$$

Furthermore,

$$
\begin{aligned}
n\left(\frac{\bar{L}}{\bar{r}^{3} \bar{p}}-\frac{L}{r^{3} p}\right) & =n\left(\frac{\bar{L} / \bar{r}^{3}-L / r^{3}}{\bar{p}}+\frac{L}{r^{3}}\left(\frac{1}{\bar{p}}-\frac{1}{p}\right)\right) \\
& \rightarrow \frac{1}{p}\left(\frac{3 L \tilde{r}}{r^{4}}-\frac{\tilde{L}}{r^{3}}\right)+\frac{L}{r^{3}} \frac{2 \tilde{w} w+\tilde{L} / r^{2}-2 \tilde{r} L / r^{3}}{2 p^{3}} .
\end{aligned}
$$

Thus most of the terms above integrate to 0 in the limit of the expression $J_{1}$, leaving us in the end with

$$
J_{1} \rightarrow\left(\mathbf{1}_{\Omega_{m}} h\right)(r, w, L) \frac{L w}{r^{3} p^{3}} \int\left(-\tilde{r} \partial_{\tilde{r}}+\tilde{w} \partial_{\tilde{w}}\right) \zeta(\tilde{r}, \tilde{w}, \tilde{L}) d \tilde{r} d \tilde{w} d \tilde{L}=0
$$

where the limit is in $L^{2}$, and 0 arises in the last equation from an integration by parts with respect to $\tilde{r}$ and $\tilde{w}$ and an exact cancellation. This completes the proof of (3.22).

The contradiction to Lemma 3.3.

The functions $h_{n}$ have all the properties required in Lemma [3.3. By Eqn. (3.22), $\lim _{n \rightarrow \infty}\left\{-E, h_{n}\right\}=\mathbf{1}_{\Omega_{m}} g$ in $L^{2}$, and since $h_{n}$ and $\mathbf{1}_{\Omega_{m}} g$ are supported in a common compact set, $\nabla U_{\left\{-E, h_{n}\right\}} \rightarrow \nabla U_{\mathbf{1}_{\Omega_{m}} g}$ in $L^{2}$ as $n \rightarrow \infty$. This implies that

$$
D^{2} \mathcal{H}_{C}\left(f_{0}\right)\left[\left\{-E, h_{n}\right\}\right] \rightarrow D^{2} \mathcal{H}_{C}\left(f_{0}\right)\left[\mathbf{1}_{\Omega_{m}} g\right], n \rightarrow \infty
$$

Since $\lim _{m \rightarrow \infty} \mathbf{1}_{\Omega_{m}} g=g$ in $L^{1} \cap L^{2}\left(\mathbb{R}^{6}\right)$, there exists $m_{0}$ sufficiently large such that $\mathbf{1}_{\Omega_{m_{0}}} g \neq 0$, and by Lemma 3.5, $\mathbf{1}_{\Omega_{m_{0}}} h \neq 0$. For all $m \geq m_{0}, \Omega_{m_{0}} \subset \Omega_{m}$ and thus $\mathbf{1}_{\Omega_{m_{0}}} h^{2} \leq \mathbf{1}_{\Omega_{m}} h^{2}$. Since $\phi^{\prime}(E)<0$ and $U_{0}^{\prime}(r)>0$ we have for all $m \geq m_{0}$,

$$
D^{2} \mathcal{H}_{C}\left(f_{0}\right)\left[\mathbf{1}_{\Omega_{m}} g\right] \geq-\frac{1}{2} \iint \frac{1}{\phi^{\prime}(E)} \frac{1}{r} U_{0}^{\prime} \mathbf{1}_{\Omega_{m_{0}}} h^{2}\left(1+|v|^{2}\right)^{-3 / 2} d v d x>0 .
$$

Letting $m \rightarrow \infty$ we get a contradiction to Lemma 3.2. and the proof of Theorem 2.1 is complete. 


\section{Global existence of the perturbed solutions}

Let $f_{0}$ be a steady state as specified in Section 2, and choose $\delta>0$ according to Theorem 2.2. For solutions with initial data $f \in \mathcal{D}_{f_{0}}$ satisfying the estimate $d\left(f, f_{0}\right)<\delta$ the potential energy and therefore also the kinetic energy remain bounded. In the present section we show that this implies that such solutions are global in time.

Proposition 4.1 Let $\stackrel{\circ}{f} \in \mathcal{D}_{f_{0}}$. Then there exists a unique, smooth solution $f$ of the relativistic Vlasov-Poisson system with $f(0)=\stackrel{\circ}{f}$ on a maximal time interval $[0, T[$ with $T>0$. If

$$
\sup _{0 \leq t<T} E_{\text {kin }}(f(t))<\infty
$$

then this solution is global, i.e., $T=\infty$.

Proof. It is well known that a compactly supported, non-negative initial datum $f \in C_{c}^{1}\left(\mathbb{R}^{6}\right)$ launches a unique $C^{1}$ solution on some time interval $[0, T[$ which we choose maximal. A detailed proof for the non-relativistic case is given in [20], and the proof carries over to the relativistic situation. In addition, the solution is known to be global if

$$
\sup \{|v| \mid(x, v) \in \operatorname{supp} f(t), 0 \leq t<T\}<\infty \text {. }
$$

However, there is a technical problem here. The steady state $f_{0}$ and therefore also data taken from $\mathcal{D}_{f_{0}}$ need not be continuously differentiable on $\mathbb{R}^{6}$, they need only be continuous on $\mathbb{R}^{6}$ and continuously differentiable on the set where they are strictly positive. But due to the spherical symmetry of the functions in $\mathcal{D}_{f_{0}}$ these regularity properties propagate to the solutions, since

$$
\nabla U(t, x)=\frac{4 \pi}{r^{2}} \int_{0}^{r} \rho(t, s) s^{2} d s \frac{x}{r}, r=|x|,
$$

is continuously differentiable if $f$ and therefore $\rho$ are continuous. In particular, the characteristic flow of the Vlasov equation is well-defined and continuously differentiable and $f$ is constant along these characteristics. To turn these arguments into a rigorous proof would require going through the local existence proof for the present situation, which would be lengthy but straight forward. 
Let us now assume a bound on the kinetic energy of a maximal, spherically symmetric solution $f$ on some time interval $[0, T$. We want to deduce a bound on the quantity

$$
P(t):=\sup \{|v| \mid(x, v) \in \operatorname{supp} f(s), 0 \leq s \leq t\},
$$

which by the continuation criterion recalled above implies global existence. The arguments which follow are adapted from [5], but we prefer to give a selfcontained presentation. First we note that a standard interpolation estimate implies that $\|\rho(t)\|_{4 / 3}$ is bounded on [0,T[, cf. [20, Lemma 5.1]. This in turn implies that for all $0 \leq s \leq t<T$,

$$
\|\nabla U(s)\|_{\infty} \leq C\|\rho(s)\|_{\infty}^{5 / 9} \leq C P^{5 / 3}(t)
$$

cf. [20, Lemma P1]; the constant $C$ depends only on the initial datum $f$ and on the bound on the kinetic energy. Due to the above formula for the field in the spherically symmetric case,

$$
\left|U^{\prime}(s, r)\right|<c \min \left\{r^{-2}, P^{5 / 3}(t)\right\} \leq c(r+a)^{-2}, r \geq 0,
$$

where we abbreviate $a:=P^{-5 / 6}(t)$; the constant $c>0$ is now kept fixed.

For $L \geq 0$ we define the function

$$
\xi_{L}(u):=\sqrt{1+u^{2}}-\frac{c u}{\sqrt{L}+a u}, u \geq 0 .
$$

It is easy to check that $\xi_{L} \in C^{2}\left(\left[0, \infty[)\right.\right.$ with $\xi_{L}^{\prime \prime}>0$. Moreover, there exists a unique $u_{L}>0$ such that $\xi_{L}\left(u_{L}\right)=1$ and $\xi_{L}$ is strictly increasing on the interval $\left[u_{L}, \infty[\right.$. Clearly,

$$
\xi_{L}(u) \geq \xi_{0}(u)=\sqrt{1+u^{2}}-\frac{c}{a}
$$

for all $u \geq 0$ and $L \geq 0$, and this implies that

$$
u_{L} \leq u_{0}=\sqrt{(1+c / a)^{2}-1}
$$

Consider now a characteristic $[0, T[\ni s \mapsto(x(s), v(s))$ in the support of $f$. With

$$
r(s):=|x(s)|, u(s):=|v(s)|, w(s):=x(s) \cdot v(s) / r(s), L(s):=|x(s) \times v(s)|^{2}
$$

it follows that $L(s)=L$ is constant and

$$
\frac{d}{d s} u^{2}(s)=-2 U^{\prime}(s, r(s)) w(s) .
$$


Let us first consider the case that $L>0$, fix some time $t \in] 0, T$, and assume that $w(t)<0$. We choose $0 \leq t_{0}<t$ minimal with the property that $w(s)<0$ for $\left.s \in] t_{0}, t\right]$. Then $u(t) \geq u\left(t_{0}\right)$, and

$$
\begin{aligned}
\frac{d}{d s}\left(\sqrt{1+u^{2}(s)}-c \int_{r(s)}^{\infty}\right. & \left.\frac{d r}{(r+a)^{2}}\right) \\
& =\left(\frac{c}{(r(s)+a)^{2}}-U^{\prime}(s, r(s))\right) \frac{w(s)}{\sqrt{1+u^{2}(s)}}<0
\end{aligned}
$$

by the estimate (4.1). Since $u^{2}(t)=w^{2}(t)+L / r^{2}(t)$ we have the estimate $r(t)>\sqrt{L} / u(t)$, and hence

$$
\sqrt{1+u^{2}\left(t_{0}\right)}-c \int_{r\left(t_{0}\right)}^{\infty} \frac{d r}{(r+a)^{2}}>\sqrt{1+u^{2}(t)}-c \int_{\sqrt{L} / u(t)}^{\infty} \frac{d r}{(r+a)^{2}}=\xi_{L}(u(t)) .
$$

If $w\left(t_{0}\right)=0$ then $u\left(t_{0}\right)=\sqrt{L} / r\left(t_{0}\right)$ and hence

$$
\xi_{L}(u(t))<\xi_{L}\left(u\left(t_{0}\right)\right),
$$

which by choice of $u_{L}$ and the fact that $u(t) \geq u\left(t_{0}\right)$ implies that $u(t) \leq u_{L} \leq$ $u_{0}$. If instead $w\left(t_{0}\right)<0$ then $t_{0}=0$ and $\xi_{L}(u(t)) \leq \sqrt{1+P^{2}(0)}$. Hence in the case $L>0$ and $w(t)<0$ we have shown that

$$
\sqrt{1+u^{2}(t)} \leq \sqrt{1+P^{2}(0)}+c / a ;
$$

notice the formula for $u_{0}$ stated above.

Next we consider the case that $L>0$ and $w(t) \geq 0$. Again, we choose $0 \leq t_{0} \leq t$ minimal such that $w(s) \geq 0$ for $s \in\left[t_{0}, t\right]$. Then $u(t) \leq u\left(t_{0}\right)$. If $t_{0}=0$ then $u(t) \leq P(0)$. If $t_{0}>0$ there must exist a sequence $0<t_{i} \nearrow t_{0}$ such that $w\left(t_{i}\right)<0$, which by the case we already dealt with implies that

$$
\sqrt{1+u^{2}\left(t_{i}\right)} \leq \sqrt{1+P^{2}(0)}+c / a,
$$

and by continuity, this estimate must also hold with $t_{0}$ instead of $t_{i}$. Hence the estimate (4.2) holds for all characteristics with $L>0$, but since the initial data of a characteristic in $\operatorname{supp} f$ with $L=0$ can be approximated as closely as we wish by data with $L>0$ and since characteristics depend continuously on their initial data the estimate (4.2) remains true if $L=0$. Hence

$$
\sqrt{1+P^{2}(t)} \leq \sqrt{1+P^{2}(0)}+c / a=\sqrt{1+P^{2}(0)}+c P^{5 / 6}(t), 0 \leq t<T .
$$

This implies that $P$ is bounded on $[0, T$ [ by some constant which depends on the initial datum $\stackrel{\circ}{f}$ and the bound for the kinetic energy, and the proof is complete. 


\section{$5 \quad$ Stationary solutions}

As we explained at the beginning of Section 2 steady states of the relativistic Vlasov-Poisson system can be obtained by making the ansatz (2.1) for some function $\phi=\phi(E)$. This ansatz solves the stationary Vlasov equation with potential $U_{0}$ in the sense that $f_{0}$ is constant along characteristics. By substituting it into the definition of the spatial density the latter becomes a functional of the potential $U_{0}$ :

$$
\rho_{0}(x)=g_{\phi}\left(U_{0}(x)\right)
$$

where

$$
g_{\phi}(u):=4 \pi \int_{u+1}^{\infty} \phi(E)(E-u)\left[(E-u)^{2}-1\right]^{1 / 2} d E, u \in \mathbb{R}
$$

Hence a steady state of the relativistic Vlasov-Poisson system is obtained, if

for a given choice of $\phi$ it can be shown that the semilinear Poisson equation

$$
\Delta U_{0}=4 \pi g_{\phi}\left(U_{0}\right)
$$

has a solution on $\mathbb{R}^{3}$ such that $f_{0}:=\phi \circ E$ has finite mass and compact support. Since in this section we consider only stationary solutions, we drop the subscript 0 and denote the steady state by $f$ and its potential by $U$.

One can show that any solution obtained by the above approach must a-posteriori be spherically symmetric, so we only look for solutions $U=$ $U(r), r \geq 0$, of the equation

$$
U^{\prime}(r)=\frac{4 \pi}{r^{2}} \int_{0}^{r} g_{\phi}(U(s)) s^{2} d s, r>0 .
$$

In 2], BATT showed that the polytropic ansatz

$$
\phi(E)=\left(E_{0}-E\right)_{+}^{k},
$$

with $k \in] 0,2[$ leads to steady states with compact support and finite mass. Below we present two approaches which can be used to prove the existence of steady states for more general functions $\phi$. First however, we show that $\phi$ must vanish for large values of $E$ if the resulting steady state is to have finite mass. 
Proposition 5.1 Let $\phi: \mathbb{R} \rightarrow[0, \infty[$ be measurable and let $(f, U)$ be a stationary solution of the relativistic Vlasov-Poisson system in the sense that $f(x, v)=\phi(E)$ and $U \in C^{1}\left(\left[0, \infty[)\right.\right.$ solves (5.3) with $g_{\phi}$ given by (5.2). Let $\iint f d v d x<\infty$. Then $U_{\infty}:=\lim _{r \rightarrow \infty} U(r)<\infty$ and $\phi(E)=0$ a.e. for $E>$ $U_{\infty}+1$.

Proof. Let $M:=\iint f d v d x$. Since by (5.3), $0 \leq U^{\prime}(r) \leq M / r^{2}, U$ is increasing with a finite limit $U_{\infty}$ for $r \rightarrow \infty$, and since $g_{\phi}$ is decreasing,

$$
M=4 \pi \int_{0}^{\infty} g_{\phi}(U(r)) r^{2} d r \geq 4 \pi \int_{0}^{\infty} g_{\phi}\left(U_{\infty}\right) r^{2} d r
$$

which means that $g_{\phi}\left(U_{\infty}\right)=0$. This is only true if $\phi(E)=0$ for almost all $E>U_{\infty}+1$.

In what follows we are only interested in steady states with finite mass so we always assume such a cut-off energy $E_{0}$.

Under suitable assumptions on $\phi$ Eqn. (5.3) has a unique solution on $[0, \infty[$, if we prescribe $U(0)$.

Proposition 5.2 Let $\phi: \mathbb{R} \rightarrow[0, \infty[$ have the following properties: $\phi$ is measurable, there exists a cut-off energy $E_{0} \in \mathbb{R}$ such that $\phi(E)=0$ for $E>E_{0}$, and there exists $k>-1$ such that for every $E_{1}<E_{0}$ there exists a constant $C \geq 0$ such that

$$
\phi(E) \leq C\left(E_{0}-E\right)^{k}, E \in\left[E_{1}, E_{0}[.\right.
$$

Then for every $u_{0} \in \mathbb{R}$ there exists a unique solution $U \in C^{1}([0, \infty[)$ of (5.3) with $U(0)=u_{0}$.

Proof. One can show that under the given assumptions on $\phi$ the function $g_{\phi}$ defined in Eqn. (5.2) is continuously differentiable. The existence and uniqueness of $U$ on some interval $[0, \delta]$ then follows by a contraction argument. Since $U$ is increasing, either $U \leq E_{0}-1$ on its maximal existence interval, and the solution is global, or there exists some $R \geq 0$ such that $U(r)>E_{0}-1$ for $r \geq R$, which implies that $g_{\phi}(U(r))=0$ for $r \geq R$, and again $U$ is global.

Remark. The solution obtained above will in general not satisfy the boundary condition $U_{\infty}=0$ which we required in previous sections, and which is part of our formulation of the relativistic Vlasov-Poisson system. However, the solution has a finite limit $U_{\infty}$, and by subtracting this limit from $U$ and redefining the cut-off energy $E_{0}$ accordingly we obtain a steady state with 
the right boundary condition. It is clear that $E_{0}$ and $U(0)$ cannot be freely prescribed under the given boundary condition for $U$ at infinity.

We now proceed to our first result which guarantees the existence of compactly supported steady states with finite mass. We follow an approach which was used for the Vlasov-Poisson and Vlasov-Einstein systems in [22].

Proposition 5.3 Let $\phi$ be as in Proposition 5.2, and assume in addition that

$$
\phi(E)=c\left(E_{0}-E\right)^{k}+O\left(\left(E_{0}-E\right)^{k+\delta}\right) \text { as } E \rightarrow E_{0}-,
$$

for some $-1 / 2<k<3 / 2, c>0$, and $\delta>0$. Let $(f, U)$ be an induced steady state of the relativistic Vlasov-Poisson system, i.e., $f(x, v)=\phi(E)$, and $U \in$ $C^{1}([0, \infty[)$ satisfies (5.3). Then this steady state has compact support and finite mass.

It is remarkable that up to technical assumptions only the behavior of $\phi$ at the cut-off energy $E_{0}$ needs to be specified. In particular, there are plenty of functions $\phi$ which satisfy the assumptions of this theorem and of the stability result in Section 2 as well. Beside the polytropic states with $1 \leq k<3 / 2$ a notable example which frequently appears in the astrophysics literature is a King type model

$$
f(x, v)=\phi(E)=\left(e^{E_{0}-E}-1\right)_{+} .
$$

The basic set-up of the proof of Proposition 5.3 is as follows. If $U(0) \geq$ $E_{0}-1$ then this relation holds also for $r>0$, and the steady state is trivial. Hence we consider a solution $U$ such that $U(0)<E_{0}-1$, and we define $[0, R[$ as the maximal interval on which $U<E_{0}-1$ so that $\operatorname{supp} \rho=[0, R]$. If $R=\infty$ then $U_{\infty}=\lim _{r \rightarrow \infty} U(r) \leq E_{0}-1$ exists by monotonicity. Now assume that $U_{\infty}<E_{0}-1$. Then $\rho(r) \geq 4 \pi g_{\phi}\left(U_{\infty}\right)>0$ which implies that $U^{\prime}(r) \geq C r$ with $C>0$ which upon integration gives the contradiction that $U_{\infty}=\infty$. Hence

$$
U(r)<E_{0}-1 \text { on }\left[0, R\left[\text {, and } \lim _{r \rightarrow R-} U(r)=E_{0}-1 ;\right.\right.
$$

if $R<\infty$ the limit assertion is true as well. In order to show that (5.4) indeed implies that $R<\infty$ we use a special case of a result due to MAKINo [15]; a proof can also be found in [22, Lemma 3.2].

Lemma 5.4 Let $x, y \in C^{1}(] 0, R[)$ be such that $x, y>0$ and

$$
\begin{aligned}
& r x^{\prime}=\alpha(r) y-x+x^{2} \\
& r y^{\prime}=y(a-\beta(r) x)
\end{aligned}
$$


on $] 0, R\left[\right.$, where $a>0$ is a constant, $\alpha, \beta \in C(] 0, R[), \inf _{r \in] 0, R[} \alpha(r)>0$, and $\left.\lim _{r \rightarrow R-} \beta(r) \in\right] 0, a[$. Then $R<\infty$.

Proof of Proposition [5.3] We first introduce the radial pressure

$$
p(x)=p(r):=\int\left(\frac{x \cdot v}{r}\right)^{2} f(x, v) d v .
$$

Like the spatial density $\rho$ the pressure becomes a functional of $U$ via Eqn. (2.1):

$$
p(r)=h_{\phi}(U(r))
$$

where

$$
h_{\phi}(u):=\frac{4 \pi}{3} \int_{u+1}^{\infty} \phi(E)(E-u)\left[(E-u)^{2}-1\right]^{3 / 2} d E, u \in \mathbb{R} .
$$

On the interval $] 0, R[$ we define

$$
\eta(r):=E_{0}-U(r)-1, m(r):=4 \pi \int_{0}^{r} s^{2} \rho(s) d s
$$

and

$$
x(r):=\frac{m(r)}{r \eta(r)}, y(r):=4 \pi r^{2} \frac{\rho^{2}(r)}{p(r)} .
$$

A short computation shows that the latter functions satisfy a system of ODEs of the form stated in Lemma 5.4 with $a:=2$ and

$$
\begin{aligned}
& \alpha:=\frac{p}{\rho \eta}=\frac{h(\eta)}{g(\eta) \eta}, \\
& \beta:=\left(\frac{r p^{\prime}}{p}-\frac{2 r \rho^{\prime}}{\rho}\right) \frac{r \eta}{m}=2 \eta \frac{g^{\prime}(\eta)}{g(\eta)}-\eta \frac{h^{\prime}(\eta)}{h(\eta)} .
\end{aligned}
$$

For the sake of convenience we have redefined

$$
\begin{aligned}
& g(\eta):=4 \pi \int_{0}^{\eta} \psi(\epsilon)(1+\eta-\epsilon)\left[2(\eta-\epsilon)+(\eta-\epsilon)^{2}\right]^{1 / 2} d \epsilon, \\
& h(\eta):=\frac{4 \pi}{3} \int_{0}^{\eta} \psi(\epsilon)(1+\eta-\epsilon)\left[2(\eta-\epsilon)+(\eta-\epsilon)^{2}\right]^{3 / 2} d \epsilon,
\end{aligned}
$$

and

$$
\psi(\epsilon):=\phi\left(E_{0}-\epsilon\right)=c \epsilon^{k}+O\left(\epsilon^{k+\delta}\right) \text { as } \epsilon=E_{0}-E \rightarrow 0+.
$$


In order to investigate the asymptotic behavior of $\beta$ we also need

$$
\begin{aligned}
& g^{\prime}(\eta)=4 \pi \int_{0}^{\eta} \psi(\epsilon)\left[\left(2(\eta-\epsilon)+(\eta-\epsilon)^{2}\right)^{-1 / 2}+2\left(2(\eta-\epsilon)+(\eta-\epsilon)^{2}\right)^{1 / 2}\right] d \epsilon, \\
& h^{\prime}(\eta)=4 \pi \int_{0}^{\eta} \psi(\epsilon)\left[\left(2(\eta-\epsilon)+(\eta-\epsilon)^{2}\right)^{1 / 2}+\frac{4}{3}\left(2(\eta-\epsilon)+(\eta-\epsilon)^{2}\right)^{3 / 2}\right] d \epsilon .
\end{aligned}
$$

We need to substitute the asymptotic expansion of $\psi$ into $g, h$, and their derivatives; note that $0<\eta(r) \rightarrow 0$ as $r \rightarrow R-$. The leading order terms always contain an integral of the form

$$
\int_{0}^{\eta} \epsilon^{k}(\eta-\epsilon)^{m} d \epsilon=\eta^{k+m+1} \int_{0}^{1} s^{k}(1-s)^{m} d s=: \eta^{k+m+1} c_{k, m}, m>-1,
$$

where the constants $c_{k, m}$ satisfy the relation

$$
\frac{c_{k, m-1}}{c_{k, m}}=\frac{k+m+1}{m}, m>0 .
$$

Hence for $\eta \rightarrow 0+$,

$$
\begin{aligned}
g(\eta) & =4 \pi c 2^{1 / 2} c_{k, 1 / 2} \eta^{k+3 / 2}+O\left(\eta^{k+3 / 2+\delta}\right), \\
h(\eta) & =\frac{4 \pi}{3} c 2^{3 / 2} c_{k, 3 / 2} \eta^{k+5 / 2}+O\left(\eta^{k+5 / 2+\delta}\right), \\
g^{\prime}(\eta) & =4 \pi c 2^{-1 / 2} c_{k,-1 / 2} \eta^{k+1 / 2}+O\left(\eta^{k+1 / 2+\delta}\right), \\
h^{\prime}(\eta) & =4 \pi c 2^{1 / 2} c_{k, 1 / 2} \eta^{k+3 / 2}+O\left(\eta^{k+3 / 2+\delta}\right) .
\end{aligned}
$$

If we substitute these expansions into the formula for $\alpha$ we find

$$
\alpha(r)=\frac{2}{3} \frac{c_{k, 3 / 2}}{c_{k, 1 / 2}}+O\left(\eta^{\delta}(r)\right) \rightarrow(k+3 / 2+1)^{-1}>0, r \rightarrow R-,
$$

Clearly, $\alpha(r)>0$ for $0<r<R$ and since $\eta(0)>0$,

$$
\lim _{r \rightarrow 0+} \alpha(r)=\frac{h(\eta(0))}{\eta(0) g(\eta(0))}>0
$$

so that $\inf _{r \in] 0, R[} \alpha(r)>0$ as desired. As to $\beta$,

$$
\beta(r)=\frac{c_{k,-1 / 2}}{c_{k, 1 / 2}}-\frac{3}{2} \frac{c_{k, 1 / 2}}{c_{k, 3 / 2}}+O\left(\eta^{\delta}(r)\right) \rightarrow k+\frac{1}{2}, r \rightarrow R-,
$$


and by the assumption on $k$ this limit lies in the interval $] 0,2[$. We can therefore apply Lemma 5.4 to conclude that $R<\infty$, and the proof of Proposition 5.3 is complete.

Although the class of steady states obtained by the approach above is quite large, the condition on $k$ excludes for example polytropes with $k \geq 3 / 2$ and possible generalizations of those. Hence we present a second approach the idea of which is the following. If one introduces the speed of light $c$ as a parameter into the relativistic Vlasov-Poisson system then solutions of this system tend to solutions of the non-relativistic case as $c \rightarrow \infty$. Exploiting this for Eqn. (5.3) one can show that the potential in the relativistic case has to cross the cut-off energy for sufficiently large $c$, if the corresponding potential in the non-relativistic case does. In this way one can show that essentially every compactly supported steady state of the non-relativistic Vlasov-Poisson system has its relativistic counterpart. In particular, this will be true for the polytropic ansatz with $0 \leq k<7 / 2$, which extends the range found in [2]. The perturbation argument outlined above was used for the Vlasov-Einstein system in 21.

If the speed of light is not normalized to unity in the relativistic VlasovPoisson system the only change is that $\sqrt{1+|v|^{2}}$ must be replaced by $\sqrt{1+|v|^{2} / c^{2}}$. In the sequel we use the parameter $\gamma:=1 / c^{2}$. In order to obtain the correct non-relativistic limit as $\gamma \rightarrow 0$ we adjust the ansatz (2.1):

$$
f(x, v)=\phi\left(\frac{1}{\gamma} \sqrt{1+\gamma|v|^{2}}+U(x)-\frac{1}{\gamma}\right) .
$$

This ansatz satisfies the Vlasov equation with potential $U$ and parameter $\gamma$. As before the spatial density $\rho$ becomes a functional of the potential $U$,

$$
\rho(r)=g_{\phi}(\gamma, U(r))
$$

where

$$
g_{\phi}(\gamma, u):=4 \pi \int_{u}^{\infty} \phi(E)(1+\gamma(E-u))\left[2(E-u)+\gamma(E-u)^{2}\right]^{1 / 2} d E, u \in \mathbb{R}
$$

and it remains to analyze the Poisson equation for $U$ which takes the form

$$
U^{\prime}(r)=\frac{4 \pi}{r^{2}} \int_{0}^{r} g_{\phi}(\gamma, U(s)) s^{2} d s, r>0
$$


For $\gamma=0$ this is precisely the equation which arises if one looks for steady states of the non-relativistic Vlasov-Poisson system via the ansatz

$$
f(x, v)=\phi\left(\frac{1}{2}|v|^{2}+U(x)\right),
$$

and an analysis of the limit $\gamma \rightarrow 0$ yields the following result.

Proposition 5.5 Let $\phi \in L_{\mathrm{loc}}^{\infty}(\mathbb{R}), \phi \geq 0$, and $\phi(E)=0, E \geq E_{0}$. Let $\left(f_{0}, U_{0}\right)$ be a non-trivial, compactly supported steady state of the Vlasov-Poisson system obtained by the ansatz (5.8), i.e., $U_{0} \in C^{1}([0, \infty[)$ solves Eqn. (5.7) with $\gamma=0$, and $U_{0}(0)<E_{0}$ but $U_{0}(R)>E_{0}$ for some $R>0$. Then for all $\gamma>0$ sufficiently small the solution $U \in C^{1}\left(\left[0, \infty[)\right.\right.$ of $(5.7)$ with $U(0)=U_{0}(0)$ satisfies $U(R)>E_{0}$, i.e., the resulting steady state of the relativistic Vlasov-Poisson system with parameter $\gamma$ has compact support and finite mass.

Proof. It is easy to see that there exists a constant $C>0$ such that

$$
\left|g_{\phi}(\gamma, u)-g\left(0, u_{0}\right)\right| \leq C \gamma+C\left|u-u_{0}\right|, u, u_{0} \geq U_{0}(0) .
$$

In what follows, constants denoted by $C$ are positive, may change from line to line, and never depend on $\gamma, U$, or $r$. By monotonicity the solutions $U$ and $U_{0}$ under consideration take only values $u \geq U_{0}(0)$, and hence

$$
\left|U^{\prime}(r)-U_{0}^{\prime}(r)\right| \leq C \gamma+C \int_{0}^{r}\left|U(s)-U_{0}(s)\right| d s, 0 \leq r \leq R .
$$

If we integrate this estimate, observe that $U(0)=U_{0}(0)$, and apply Gronwall's lemma we find that

$$
\left|U(r)-U_{0}(r)\right| \leq C \gamma, 0 \leq r \leq R
$$

The latter holds in particular for $r=R$, and since by assumption $U_{0}(R)>E_{0}$ the same is true for $U$, provided $\gamma$ is small enough.

Remark. The requirement that $\gamma$ must be sufficiently small in the result above actually changes the relativistic Vlasov-Poisson system we started with. However, it is easy to check that if $f$ is a steady state of the system with some $\gamma>0$ then the rescaled function $f_{\gamma}(x, v):=\gamma^{-3 / 2} f\left(\gamma^{-1 / 2} x, \gamma^{-1 / 2} v\right)$ is a steady state of the original system with $\gamma=1$. This scaling changes the cut-off energy, which however has to be adjusted in order to satisfy the boundary condition $U(\infty)=0$ anyway, and it changes the ansatz, 
$f_{\gamma}(x, v)=\gamma^{-3 / 2} \phi\left(\left(\sqrt{1+|v|^{2}}+U(x)-1\right) / \gamma\right)$, but it does not change the principal way in which the ansatz depends on the particle energy.

In the non-relativistic case it is well known that the polytropic ansatz with $0 \leq k<7 / 2$ leads to compactly supported steady states; as a matter of fact the compact support is lost for $k=7 / 2$, the so-called Plummer sphere, and finite mass is lost for $k>7 / 2$. Generalizations of these polytropes were obtained by variational techniques in [10, and by techniques which are closer to the ones used to prove Proposition [5.3 in [11], so there are many examples which one can extend to the relativistic case by Proposition 5.5 .

To conclude this section we emphasize that its purpose was only to demonstrate that there are many examples of steady states which satisfy the assumptions of our stability analysis. Its purpose was not a systematic investigation of the possible steady states of the relativistic Vlasov-Poisson system.

Acknowledgment. It is a pleasure for the authors to thank Prof. Y. Guo for helpful discussions on the subject of this paper.

\section{References}

[1] H. Andréasson: The Einstein-Vlasov system / Kinetic Theory. Living Reviews in Relativity. Published by the Max Planck Institute for Gravitational Physics, http://www.livingreviews.org/lrr-2005-2

[2] Batt, J.: Steady state solutions of the relativistic Vlasov-Poisson system. Proceedings of the Fifth Marcel Grossmann Meeting on General relativity, Part A, B (Perth, 1988), 1235-1247, World Sci. Publishing, Teaneck, NJ, 1989

[3] Binney, J., Tremaine, S.: Galactic Dynamics. Princeton: Princeton University Press 1987

[4] Fridman, A., Polyachenko, V.: Physics of Gravitating Systems I. New York: Springer-Verlag 1984

[5] Glassey, R. T., Schaeffer, J.: On symmetric solutions of the relativistic Vlasov-Poisson system. Comm. Math. Phys. 101, 459-473 (1985)

[6] Guo, Y.: Variational method in polytropic galaxies. Arch. Ration. Mech. Anal. 150, 209-224 (1999) 
[7] Guo, Y.: On the generalized Antonov's stability criterion. Contemp. Math. 263, 85-107 (2000)

[8] Guo, Y., Rein, G.: Stable steady states in stellar dynamics. Arch. Ration. Mech. Anal. 147, 225-243 (1999)

[9] Guo, Y., Rein, G.: Isotropic steady states in galactic dynamics. Comm. Math. Phys. 219, 607-629 (2001)

[10] Guo, Y., Rein, G.: A non-variational approach to nonlinear stability in stellar dynamics applied to the King model. Preprint, 2006

[11] Heinzle, M., Rendall, A., Uggla, C.: Theory of Newtonian selfgravitating stationary spherically symmetric systems. Math. Proc. Camb. Phil. Soc. 140, 177-192 (2006).

[12] Kandrup, H., Sygnet, J. F.: A simple proof of dynamical stability for a class of spherical clusters. Astrophys. J. 298, 27-33 (1985)

[13] Lemou, M., Mehats, F., Raphael, P.: On the orbital stability of the ground states and the singularity formation for the gravitational VlasovPoisson system. Preprint 2005

[14] Lions, P.-L., Perthame, B.: Propagation of moments and regularity for the 3-dimensional Vlasov-Poisson system. Invent. Math. 105, 415-430 (1991)

[15] Makino, T.: On spherically symmetric stellar models in general relativity. J. of Math. of Kyoto University 38, 55-69 (1998)

[16] Perez, J., Aly, J.-J.: Stability of spherical stellar systems I: Analytical results. Mon. Not. R. Astron. Soc. 280, 689-699 (1996)

[17] Pfaffelmoser, K.: Global classical solutions of the Vlasov-Poisson system in three dimensions for general initial data. J. Differential Equations 95, 281-303 (1992)

[18] Rein, G.: Reduction and a concentration-compactness principle for energy-Casimir functionals. SIAM J. Math. Anal. 33, 896-912 (2002) 
[19] Rein, G.: Nonlinear stability of Newtonian galaxies and stars from a mathematical perspective. In: Nonlinear Dynamics in Astronomy and Physics, Annals of the New York Academy of Sciences 1045, 103-119 (2005)

[20] Rein, G.: Collisionless Kinetic Equations from Astrophysics-The Vlasov-Poisson system. Handbook of Differential Equations, Evolutionary Equations. Vol. 3. Eds. C. M. Dafermos and E. Feireisl, Elsevier, to appear

[21] Rein, G., Rendall, A.: Smooth static solutions of the spherically symmetric Vlasov-Einstein system. Ann. Inst. Henri Poincare 59, 383-397 (1993)

[22] Rein, G., Rendall, A.: Compact support of spherically symmetric equilibria in non-relativistic and relativistic galactic dynamics. Math. Proc. Camb. Phil. Soc. 128, 363-380 (2000)

[23] Schaeffer, J.: Global existence of smooth solutions to the Vlasov-Poisson system in three dimensions Comm. Partial Differential Equations 16, 1313-1335 (1991)

[24] Sygnet, J. F., Des Forets, G., Lachieze-Rey, M., Pellat, R.: Stability of gravitational systems and gravothermal catastrophe in astrophysics. Astrophys. J. 276, 737-745 (1984) 